TRANSACTIONS OF THE

AMERICAN MATHEMATICAL SOCIETY

Volume 349, Number 4, April 1997, Pages 1389-1420

S 0002-9947(97)01565-1

\title{
GROUP ACTIONS ON ARRANGEMENTS OF LINEAR SUBSPACES AND APPLICATIONS TO CONFIGURATION SPACES
}

\author{
SHEILA SUNDARAM AND VOLKMAR WELKER
}

\begin{abstract}
For an arrangement of linear subspaces in $\mathbb{R}^{n}$ that is invariant under a finite subgroup of the general linear group $G l_{n}(\mathbb{R})$ we develop a formula for the $G$-module structure of the cohomology of the complement $\mathcal{M}_{\mathcal{A}}$. Our formula specializes to the well known Goresky-MacPherson theorem in case $G=1$, but for $G \neq 1$ the formula shows that the $G$-module structure of the complement is not a combinatorial invariant. As an application we are able to describe the free part of the cohomology of the quotient space $\mathcal{M}_{\mathcal{A}} / G$. Our motivating examples are arrangements in $\mathbb{C}^{n}$ that are invariant under the action of $S_{n}$ by permuting coordinates. A particular case is the " $k$-equal" arrangement, first studied by Björner, Lovász, and Yao motivated by questions in complexity theory. In these cases $\mathcal{M}_{\mathcal{A}}$ and $\mathcal{M}_{\mathcal{A}} / S_{n}$ are spaces of ordered and unordered point configurations in $\mathbb{C}^{n}$ many of whose properties are reduced by our formulas to combinatorial questions in partition lattices. More generally, we treat point configurations in $\mathbb{R}^{d}$ and provide explicit results for the " $k$-equal" and the " $k$-divisible" cases.
\end{abstract}

\section{INTRODUCTION}

In this paper we develop combinatorial and representation-theoretic methods in the theory of arrangements of linear subspaces in $\mathbb{R}^{d}$, which can be applied to the study of the cohomology of spaces of ordered and unordered point configurations in $d$-dimensional real space. These spaces arise in various contexts in mathematics. Although the relation between hyperplane arrangements and configuration spaces had been established a long time ago by Fadell and Neuwirth [Fa-Ne], the idea of using subspace arrangements as a unifying approach in this context seems to be recent. The connection between arrangements of linear subspaces and configuration spaces was first suggested by Björner $[\mathrm{Bj} 4,8,5]$, who was himself inspired by the work of Arnol'd [Ar1], [Ar2], [Ar3] and Vassiliev [Va2]. In Björner's orginal motivation $[\mathrm{Bj}-\mathrm{Lo}-\mathrm{Y}]$ ordered point configurations were studied in the context of lower bounds in computational complexity. In a subsequent development Björner and Lovász [Bj-Lo] showed that the cohomology of spaces of ordered point configurations provides even better lower bounds in the linear decision tree model. Parallel to that development, Vassiliev [Va1] used order complexes of partition lattices as building blocks in the construction of his knot invariant. Exactly these partition

Received by the editors January 1, 1965 .

1991 Mathematics Subject Classification. Primary 05E25, 57N65. Secondary 20C30, 55M35.

Key words and phrases. Subspace arrangements, group action, poset homology, configuration spaces, homotopy limits, symmetric functions.

The author acknowledges support by the DFG while he was visiting scholar at MIT. 
lattices and their generalizations, considered by Vassiliev [Va2], [Va3] and Björner and Welker [Bj-We], serve also as the ingredients in the study of ordered configuration spaces carried out in this paper (see also [Me] for related construction of invariants of ornaments). The orbit spaces of the ordered configuration spaces under the action of the symmetric group are, in case of point configurations in $\mathbb{C}^{n}$, spaces of monic polynomials of degree $n$ with certain restrictions on the root multiplicities. Examples of such spaces have been studied by Arnol'd [Ar1], [Ar2], [Ar3], Brieskorn [Bri], Fox and Neuwirth [Fo-Ne], Fuks [Fu] and Vassiliev [Va2]. We provide and apply general tools for determining representations of finite groups on the rational cohomology of the complement of an arrangement of linear subspaces. This will enable us to make some general statements on the integral cohomology of ordered configuration spaces and the rational cohomology of unordered configuration spaces. More precise information will be provided for the following examples:

(A) Let $\mathcal{M}_{\left(k, 1^{q}\right)}^{d}$ be the set of all $n=k+q$ tuples $\left(x_{1}, \ldots, x_{n}\right)$ of points $x_{i} \in \mathbb{R}^{d}$ such that there is no subset $E$ of $\{1, \ldots, n\}$ of cardinality $k$ satisfying $x_{t}=x_{s}$ for all $t, s \in E$. In particular $\mathcal{M}_{\left(k, 1^{q}\right)}^{d}$ is the pure braid space (see [Fa-Ne], [Ar1]) for $k=d=2$. (See [Co-La-Ma] for $k=2$ and $d \geq 2$. For general $k$ the space $\mathcal{M}_{\left(k, 1^{q}\right)}^{d}$ is first mentioned in $[\mathrm{Co}-\mathrm{Lu}]$ in connection with a generalisation of the Borsuk-Ulam Theorem.) The cohomology of the spaces $\mathcal{M}_{\left(k, 1^{q}\right)}^{d}$ was determined in [Bj-We] for $d=1,2$ (and implicitly for general $d$ ). We study the action of the symmetric group $S_{n}$ on $\mathcal{M}_{\left(k, 1^{q}\right)}^{d}$ by permuting the coordinates. For $d=2$ the orbit space $\mathcal{M}_{\left(k, 1^{q}\right)}^{d} / S_{n}$ is homeomorphic to the space of monic polynomials of degree $n$ with no root of multiplicity $k$. For $k=2$, this is the complement of the discriminant; it is thus the braid space for the braid group on $n$ strings (see [Fo-Ne], [Fu] for the basic results on this space). For general $k$ this space was studied by Arnol'd [Ar2], who computed the integral cohomology of $\mathcal{M}_{\left(k^{r}, 1^{q}\right)}^{2} / S_{n}$ (see paragraph (B) below for definitions in the case $r \neq 1$ ). We determine the representation of $S_{n}$ on the rational cohomology of $\mathcal{M}_{\left(k, 1^{q}\right)}^{d}$. For the pure braid space, this was done by Lehrer and Solomon ([Le-So]). By calculating the multiplicity of the trivial character in this representation, we compute the rational cohomology of $\mathcal{M}_{\left(k, 1^{q}\right)}^{d} / S_{n}$ and hence retrieve the result of Arnol'd on the rational cohomology of $\mathcal{M}_{\left(k^{r}, 1^{q}\right)}^{2} / S_{n}$ for $r=1$.

(B) More generally, for a partition $\lambda=\left(\lambda_{1} \geq \cdots \geq \lambda_{n}\right) \vdash n$ of the integer $n$ we study the set $\mathcal{M}_{\lambda}^{d}$ of $n$-tuples in $\mathbb{R}^{d}$ satisfying the condition that there is no partition of $\{1, \ldots, n\}$ into blocks $E_{i}$ of size $\lambda_{i}$ such that for all $i$ and all $s, t \in E_{i}$ we have $x_{s}=x_{t}$. These spaces have been introduced by Björner; they occur as complements of the orbit arrangements discussed in [Bj4]. As mentioned in paragraph (A), for $\lambda=\left(k^{r}, 1^{q}\right)$ and $d=2$ the integral cohomology of the orbit space $\mathcal{M}_{\left(k^{r}, 1^{q}\right)}^{d} / S_{n}$ was determined by Arnol'd in [Ar2]. We determine some general results on $\mathcal{M}_{\lambda}^{d}$ and its orbit space $\mathcal{M}_{\lambda}^{d} / S_{n}$. We give the Euler characteristic and determine some facts about the distribution of rational cohomology. These results lead us to a conjecture generalising the so-called "Finiteness" condition of Arnol'd [Ar2]. As a second specific example, we determine the representation on the rational cohomology of $\mathcal{M}_{\lambda}^{d}$ for $\lambda=\left(k^{\ell}\right)$, and all $d \geq 1$; in particular we recover the result of Arnol'd for the unordered configuration space $\mathcal{M}_{\lambda}^{2} / S_{n}$.

In the sequel we outline the approach used in this paper in order to obtain results on the spaces described in (A)-(B). The basic ideas of this approach can be found in Björner's survey article $[\mathrm{Bj} 4,8.5]$. Before we can formulate the approach we 
shall have to introduce some notation. By an arrangement $\mathcal{A}$ of linear subspaces in $\mathbb{R}^{n}$ we understand a finite set of linear subspaces in $\mathbb{R}^{n}$ which is closed under intersection (i.e. for all $x, y \in \mathcal{A}$ we have $x \cap y \in \mathcal{A}$ ). If $G$ is a finite subgroup of $G l_{n}(\mathbb{R})$ then we call an arrangement $\mathcal{A}$ a $G$-arrangement if for all $V \in \mathcal{A}$ and $g \in G$ the image $V^{g}$ lies also in $\mathcal{A}$. By $L_{\mathcal{A}}$ we denote the set $\mathcal{A}$ ordered by reversed inclusion and enlarged by the additional least element $\mathbb{R}^{n}$. By $\hat{0}$ (resp. $\hat{1}$ ) we denote the least (resp. greatest) element in $L_{\mathcal{A}}$. The partially ordered set $L_{\mathcal{A}}$ is in fact a lattice, the intersection lattice of the arrangement $\mathcal{A}$. In general we call a finite partially ordered set $P$ a poset. Of particular interest for our work is the order complex $\Delta(P)$ of the poset $P$ with least element $\hat{0}$ and greatest element $\hat{1}$. The simplices in $\Delta(P)$ are the linearly ordered subsets of $P$, which do NOT contain $\hat{0}$ or $\hat{1}$. In particular $\Delta(P)$ is a simplicial complex. In our situation we have to study the case where $P$ is an interval, $P=[\hat{0}, x]=\{y \mid \hat{0} \leq y \leq x\}$, in the intersection lattice $L_{\mathcal{A}}$ of the arrangement $\mathcal{A}$.

The link $D_{\mathcal{A}}$ of $\mathcal{A}$ is the intersection of the set-theoretic $\bigcup_{V \in \mathcal{A}} V$ of the arrangement with the unit sphere $S^{n-1}$ in $\mathbb{R}^{n}$. In case $\mathcal{A}$ is a $G$-arrangement we can always assume that $S^{n-1}$ is the space of points of unit distance with respect to a $G$-invariant scalar product $\left\langle\underline{\mathbf{x}_{1}}, \underline{\mathbf{x}_{2}}\right\rangle_{G}=1 /|G| \cdot \sum_{g \in G}\left\langle g \cdot \underline{\mathbf{x}_{1}}, g \cdot \underline{\mathbf{x}_{2}}\right\rangle$ and is therefore also $G$-invariant (here $\langle$,$\rangle denotes the standard scalar product in \mathbb{R}^{n}$ ). The complement $\mathcal{M}_{\mathcal{A}}$ of the arrangement $\mathcal{A}$ is the complement of the union of $\mathcal{A}$ in $\mathbb{R}^{n}$. Note that if $\mathcal{A}$ is a $G$-arrangement then $G$ acts on $D_{\mathcal{A}}$ and $\mathcal{M}_{\mathcal{A}}$ as a group of homeomorphisms. In particular the homology and cohomology groups of $D_{\mathcal{A}}$ and $\mathcal{M}_{\mathcal{A}}$ become $G$-modules. Moreover $G$ acts on $L_{\mathcal{A}}$ as a group of lattice automorphisms. Since it is easily seen that the spaces described in (A) and (B) are complements of suitably chosen arrangements of linear subspaces, we can now formulate our approach using the notation introduced above:

(STEP 1) In Section 2 we provide a combinatorial tool for the study of the representation of a finite group $G \leq G l_{n}(\mathbb{R})$ on the complement $\mathcal{M}_{\mathcal{A}}$ of a $G$ invariant arrangement $\mathcal{A}$ of linear subspaces in $\mathbb{R}^{d}$. The formula of Goresky and MacPherson [Go-MacP] describes the cohomology of $\mathcal{M}_{\mathcal{A}}$ in terms of the homology of order complexes of lower intervals in the intersection lattice $L_{\mathcal{A}}$. In Theorem 2.5 (ii) of this paper, we generalise the Goresky-MacPherson result to an isomorphism of $G$-modules. It turns out that this representation is not completely encoded by the intersection lattice of the arrangement; it is necessary, in addition, to consider the action of various subgroups of $G$ on the homology of certain spheres. These one-dimensional representations will, however, be easy to determine. Moreover our results allow us to make general statements about orbit spaces of complements of subspace arrangements. Our proof of the G-equivariant Goreksy-MacPherson formula uses tools from the theory of diagrams of spaces ([Bo-Ka], [tDi], [Vo]), first applied in this context by Ziegler and Živaljević [Zie-Ž]. In contrast to their work we cannot use homotopy-methods in this $G$-equivariant setting, but have to resort to a spectral sequence in the semisimplicial category introduced by Segal [Se]. Other proofs of the "standard" Goresky-MacPherson formula can be found in [Hu], [Je] and $[\mathrm{Va} 2]$.

(STEP 2) From the work of Sundaram [Su] and Sundaram and Wachs [Su-Wa] we obtain a description of the representation of $G=S_{n}$ on the homology of the 
intersection lattices $L_{\mathcal{A}_{\left(k, 1^{n-k}\right)}^{d}}$ and $L_{\mathcal{A}_{\left(k^{\ell}\right)}^{d}}$. We also find there the computation of the representation of $G_{x}$ on the homology of the lower intervals $[\hat{0}, x]$ in both cases. By the Goresky-MacPherson formula for $G$-modules this suffices to pursue (STEP $3)$.

(STEP 3) In Section 3 we determine the multiplicity $c_{i}$ of the trivial representation on the $i$ th cohomology group of the complement; these calculations are considerably simplified by working in the ring of symmetric functions. By wellknown facts [Bre], it follows that the $i$ th cohomology group of $\mathcal{M}_{\mathcal{A}} / G$ is a free $\mathbb{Q}$-module of rank $c_{i}$.

In order to carry out our approach for more general classes of arrangements and configuration spaces, one has to prove results similar to those in $[\mathrm{Su}]$ and $[\mathrm{Su}-\mathrm{Wa}]$ for the relevant lattices. This requires a thorough understanding of the intersection lattice $L_{\mathcal{A}}$ of the arrangement, both topologically and combinatorially. For the spaces in cases (A) and (B) the intersection lattice $L_{\mathcal{A}}$ of the corresponding arrangement is always naturally embedded as a join sublattice in the lattice of set partitions $\Pi_{n}$ of the set $\{1, \ldots, n\}$.

For an integer partition $\lambda \vdash n$ of $n$, the manifold $M_{\lambda}^{d}$ is the complement of an arrangement $\mathcal{A}_{\lambda}^{d}$ in $\mathbb{R}^{d}$ whose intersection lattice $L_{\mathcal{A}_{\lambda}^{d}}$ is isomorphic to the joinsublattice $\Pi_{\lambda}$ of $\Pi_{n}$ generated by all partitions $\pi$ of type $\lambda$. The type of a set partition $\pi$ is the integer partition of $n$ arising from the set of block sizes of $\pi$.

- The " $k$-equal" lattice $\Pi_{\left(k, 1^{n-k}\right)}$ was first considered in [Bj-Lo-Y] (denoted there by $\left.\Pi_{n, k}\right)$ and $[\mathrm{Bj}-\mathrm{Lo}]$. It was then investigated by Björner and Welker, who showed in [Bj-We] that any lower interval in $\Pi_{\left(k, 1^{n-k}\right)}$ has the homotopy type of a wedge of spheres, and determined the Betti numbers. In [Su-Wa] Sundaram and Wachs determine the characteristic of the $S_{n^{-}}$ representation on the homology of $\Pi_{\left(k, 1^{n-k}\right)}$. For general $\lambda \vdash n$ the lattices $\Pi_{\lambda}$ were introduced in $[\mathrm{Bj} 4]$ as the intersection lattices of Björner's orbit arrangements.

- The $k$-divisible lattice $\Pi_{\left(k^{\ell}\right)}$ is the intersection lattice of the orbit arrangement corresponding to the partition $\left(k^{\ell}\right)$ of $n$ ([Bj4, Example 3.3]). This lattice has been studied by various authors ([C-H-R], [Sa], [Wa]). In particular it follows from [Sa] and [Wa] that $\Delta\left(\Pi_{\left(k^{\ell}\right)}\right)$ is a Cohen-Macaulay space, and the Betti numbers are known ([St1]). We carry out (STEP 3) for the manifolds $M_{\lambda}^{d}$ for $\lambda=\left(k, 1^{n-k}\right)$ and $\lambda=\left(k^{\ell}\right)$.

For a given poset $P$ our reduction (STEP 1 ) transforms all problems into problems regarding the reduced simplicial homology group $\widetilde{H}_{*}(\Delta([\hat{0}, x]))$ of the order complex of a lower interval $[\hat{0}, x]$ in $P$. For the sake of a concise notation we shall write $\widetilde{H}_{*}(\hat{0}, x)$ for $\widetilde{H}_{*}(\Delta([\hat{0}, x]))$ and $\widetilde{H}_{*}(P)$ for $\widetilde{H}_{*}(\Delta(P))$, and similarly, $\Delta(\hat{0}, x)$ for the order complex $\Delta([\hat{0}, x])$. For the rest of this paper we assume that all simplicial (resp. singular) homology is taken with COEFFICIENTS IN THE FIELD $\mathbb{Q}$ OF RATIONAL NUMBERS.

There are other interesting configuration spaces which arise as orbits of complements of subspace arrangements (see for example the spaces treated by Arnol'd [Ar3], Epshtein [Ep], Vassiliev [Va2, Chapter III][ and Guest, Kozlowski, and Yamaguchi $[\mathrm{Gu}-\mathrm{Ko}-\mathrm{Y}])$. The ordered cases and the corresponding cohomology representations do not seem to have been considered in the references given above. Moreover although the torsion-free part of the integral cohomology of the orbit 
spaces appears to be "the easy part" there does not seem to be a general approach for this problem. We therefore propose the approach outlined in (STEP 1)-(STEP 3) as a general method for tackling such problems.

We would like to thank A. Björner for inspiring the research program of this paper, and A. Björner and M. Wachs for many discussions. We also thank V.I.

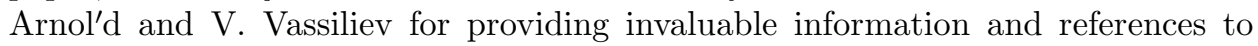
their work.

\section{2. $(P, G)$-Diagrams And a Goresky-MacPherson Formula FOR $G$-MODULES}

In this section we extend the formula of Goresky and MacPherson [Go-MacP] for the cohomology of the complement of a subspace arrangement $\mathcal{A}$ to cover also the $G$-module structure of the cohomology of the complement of a $G$-arrangement. As a special case we obtain a result of Orlik and Solomon [O-So] on complements of complex hyperplane arrangements. Our results are more general and avoid the use of de Rham's cohomology. On the other hand we are unable to determine the structure of the cohomology algebra by our methods.

Our approach to the Goresky-MacPherson formula follows the proof of the formula given by Ziegler and Živaljević [Zie-Ž]. We shall outline an equivariant approach, in which the first steps are based on their work. We omit the proofs, which are simple adaptations of the ones given by Ziegler and Živaljević. One can also use a Mayer-Vietoris spectral sequence, taking into account the group action (see [Je] for this approach in the non-equivariant case). However, our approach has the advantage that it provides more insight into homotopy.

First we introduce the notion of a $(P, G)$-diagram. Let $X$ be a space on which the group $G$ acts as a group of homeomorphisms. In general we call a space $X$ on which a group $G$ acts by homeomorphisms a $G$-space. (We define similarly the notion of a $G$-set and a $G$-poset.) Assume there exists a sequence $\left(\mathcal{D}_{x}\right)_{x \in P}$ of open and closed subspaces indexed by a $G$-set $P$ such that:

(i) $\mathcal{D}_{x} \cap \mathcal{D}_{y}=\emptyset$ for $x \neq y$.

(ii) $\left(\mathcal{D}_{x}\right)^{g}=\mathcal{D}_{x^{g}}$.

(iii) $\bigcup_{x \in P} \mathcal{D}_{x}=X$

Now assume further that $P$ is a $G$-poset ordered by " $\leq$ " and that for all $x<y$ in $P$ there is a mapping $d_{x y}: \mathcal{D}_{y} \rightarrow \mathcal{D}_{x}$ such that $d_{x y}(z)^{g}=d_{x^{g} y^{g}}\left(z^{g}\right)$. Then we call $\mathcal{D}$ a $(P, G)$-diagram. In case $G=1$, a $(P, G)$-diagram $\mathcal{D}$ is a functor from the poset $P$ (regarded as a small category) to the category of topological spaces ([Bo-Ka], [Se], [Vo], [Zie-Ž] $)$. A $(P, G)$-diagram can also be interpreted as a functor from a suitable category (here the semidirect product of $P$ and $G$ ) to the category of topological spaces, but we do not want to emphasize this point of view, since we are interested in concrete examples. In this paper we shall be concerned with the following example of $(P, G)$-diagrams. Let $X$ be the link $D_{\mathcal{A}}$ of a $G$-arrangement $\mathcal{A}$ of linear subspace in $\mathbb{R}^{n}$. For each $x \in P:=L_{\mathcal{A}}-\{\hat{0}\}$ let $\mathcal{D}_{x}$ be the space $S_{x}^{\operatorname{dim}(x)-1}=S^{n-1} \cap x$. For $x<y$ the mapping $d_{x y}$ is the inclusion from $\mathcal{D}_{y}$ into $\mathcal{D}_{x}$. One immediately verifies that the conditions (ii)-(iii) are satisfied. Let us denote by $\coprod$ the sum of two topological spaces. We set $X^{\prime}=\coprod_{x \in \mathcal{A}} S_{x}^{\operatorname{dim}(x)-1}$; then $X^{\prime}$ can be equipped with a $G$-action in the following way. For a subgroup $H$ of a group $G$ and a $H$-space $Y$, 
we denote by $G \times_{H} Y$ the $G$-space induced from the $H$-space $Y$. More precisely, this is the orbit space $(G \times Y) / H$ of the $(G \times H)$-space $G \times Y$, where $(g, h) \in G \times H$ maps $\left(g^{\prime}, y\right) \in G \times Y$ to $\left.\left(g g^{\prime} h^{-1}, y^{h}\right)[\mathrm{tDi},(4.2)]\right)$. In the sequel we shall use the fact that by the functoriality of this construction there are isomorphisms of $G$-modules $\widetilde{H}^{*}\left(G \times_{H} Y\right) \cong \operatorname{Ind}_{H}^{G} \widetilde{H}^{*}(Y)$ and $\widetilde{H}_{*}\left(G \times_{H} Y\right) \cong \operatorname{Ind}_{H}^{G} \widetilde{H}_{*}(Y)$.

Now let $G_{x}$ be the stabiliser of the subspace $x$ in the arrangement $\mathcal{A}$. The identification of $X^{\prime}$ with $\coprod_{x \in \mathcal{A} / G} G \times_{G_{x}} S_{x}^{\operatorname{dim}(x)-1}$ makes $X^{\prime}$ into a $G$-space. If for $x \in \mathcal{A}$ and $g \in G$ we set $\mathcal{D}_{x^{g}}^{\mathcal{A}}=\left(g, S_{x}^{\operatorname{dim}(x)-1}\right) \subseteq X^{\prime}$, then $\mathcal{D}^{\mathcal{A}}$ satisfies conditions (i)-(iii). The natural inclusions $d_{x y}^{\mathcal{A}}$ for $x<y$ satisfy $d_{x y}^{\mathcal{A}}(z)^{g}=d_{x^{g} y^{g}}^{\mathcal{A}}\left(z^{g}\right)$. Let $\mathcal{A} / G$ be a set of representatives of the orbits of $G$ on $\mathcal{A}$, and denote by $G_{x}$ the stabiliser of $x \in \mathcal{A}$. Then the $G$-action on $\coprod_{x \in \mathcal{A} / G} G \times_{G_{x}} S_{x}^{\operatorname{dim}(x)-1}$ makes $X^{\prime}$ and $\left(\mathcal{D}_{x}^{\mathcal{A}}\right)_{x \in P}$ a pair which satisfies conditions (i)-(iii). Therefore $\mathcal{D}^{\mathcal{A}}$ is a $(P, G)$-diagram. Moreover the $(P, G)$-diagram $\mathcal{D}^{\mathcal{A}}$ satisfies the additional assumption:

(HOM) For any element $x$ of $P$ the induced mapping

$$
\bigcup_{x<y} d_{x y}^{*}: \widetilde{H}_{*}\left(\bigcup_{x<y} \mathcal{D}_{y}\right) \rightarrow \widetilde{H}_{*}\left(\mathcal{D}_{x}\right)
$$

is trivial.

This follows from the fact that in this case the image of $\bigcup_{x<y} \mathcal{D}_{y}$ is a $k$-dimensional complex embedded in the $(\operatorname{dim}(x)-1)$-connected space for $k \leq \operatorname{dim}(x)-1$. But this mapping is Nот $G$-homotopically trivial in general. Assume for example that the cyclic group $C_{2}$ of order two acts on the standard 1 -sphere in $\mathbb{R}^{2}$ by the reflection along a line through the origin. Then the embedded 0 -sphere (i.e. the intersection of the line with $S^{1}$ ) is not $C_{2}$-contractible in $S^{1}$, since this would change the homotopy type of the space of $C_{2}$-invariant points. This is indeed the reason why, as mentioned above, we cannot reformulate Theorem 2.4 by homotopy methods. There seems to be no easy generalisation of the Wedge Lemma of [Zie-Ž]. More generally let $\mathcal{A}=\left\{\mathcal{D}_{x} \mid x \in P\right\}$ be a set (or arrangement) of closed subcomplexes of a CWcomplex X (we need that inclusions of subspaces are cofibrations), which is closed under taking intersections. Assume further that $X$ is a $G$-space and that $\mathcal{A}$ is invariant under $G$. Let $P$ be the poset equipped with reverse inclusion as a partial order. Via the construction described above, the set of subspaces gives rise to a $(P, G)$-diagram $\mathcal{D}^{\mathcal{A}}$. But in general $\mathcal{D}^{\mathcal{A}}$ fails to satisfy (HOM). For a poset $P$ we denote by $\hat{P}$ the poset $P$ enlarged by an artificial least element $\hat{0}$ and a largest element $\hat{1}$.

We assume from now on, that all topological spaces occurring in the paper are CW-COMPLEXES.

To each $(P, G)$-diagram $\mathcal{D}$ there is an associated space $\|\mathcal{D}\|$, which is the quotient space of the space $\coprod_{x \in P} \Delta\left(\widehat{P_{\leq x}}\right) \times \mathcal{D}_{x}$ by a relation "三", defined as follows. Let $Y$ be the set $\coprod_{x<y} \Delta\left(\widehat{P_{\leq x}}\right) \times \mathcal{D}_{y}$. For a space $\Delta\left(\widehat{P_{\leq x}}\right) \times \mathcal{D}_{y}$ we denote by $\alpha: \Delta\left(\widehat{P_{\leq x}}\right) \times$ $\mathcal{D}_{y} \rightarrow \Delta\left(\widehat{P_{\leq y}}\right) \times \mathcal{D}_{y}$ the mapping induced in the first component by the inclusion of $\Delta\left(\widehat{P_{\leq x}}\right)$ into $\Delta\left(\widehat{P_{\leq y}}\right)$ and in the second component by the identity on $\mathcal{D}_{y}$. By 
$\beta: \Delta\left(\widehat{P_{\leq x}}\right) \times \mathcal{D}_{y} \rightarrow \Delta\left(\widehat{P_{\leq x}}\right) \times \mathcal{D}_{x}$ we denote the mapping induced in the first component by the identity on $\mathcal{D}_{x}$ and in the second component by the mapping $d_{x y}$. Now the relation " $\equiv$ " is generated by $\alpha(u, v) \equiv \beta(u, v)$. The space $\coprod_{x \in P} \Delta\left(\widehat{P_{\leq x}}\right) \times \mathcal{D}_{x}$ carries in a natural way the structure of a $G$-space. Namely, let $P / G$ be a set of representatives of the orbits of $G$ on the $G$-set $P$. Then $\coprod_{x \in P} \Delta\left(\widehat{P_{\leq x}}\right) \times \mathcal{D}_{x}$ is $G$ homeomorphic to $\coprod_{x \in P / G} G \times_{G_{x}}\left(\Delta\left(\widehat{P_{\leq x}}\right) \times \mathcal{D}_{x}\right)$. Analogously $\coprod_{x<y} \Delta\left(\widehat{P_{\leq x}}\right) \times \mathcal{D}_{y}$ carries a $G$-operation via its identification with $\coprod_{x<y \in P / G} G \times_{G_{x, y}}\left(\Delta\left(\widehat{P_{\leq x}}\right) \times \mathcal{D}_{y}\right)$. Here $G_{x, y}$ denotes the stabiliser of the elements $x$ and $y$ (i.e. the intersection of $G_{x}$ and $G_{y}$ ). The construction immediately implies $(a, b) \equiv(c, d) \Leftrightarrow\left(a^{g}, b^{g}\right) \equiv\left(c^{g}, d^{g}\right)$ for all $g \in G$. In particular the space $\|\mathcal{D}\|$ is a $G$-space.

Lemma 2.1. (Equivariant Projection Lemma [Zie-Ž], [Se], [Bo-Ka, XII.3.1(iv)]) Let $\mathcal{A}$ be a $G$-arrangement of subspaces of the $G$-space $X$. Let $P$ be the intersection poset of $\mathcal{A}$ and let $\mathcal{D}^{\mathcal{A}}$ be the corresponding $(P, G)$-diagram. Then the union of the subspaces in the arrangement $\mathcal{A}$ and the homotopy limit $\left\|\mathcal{D}^{\mathcal{A}}\right\|$ are $G$-homotopy equivalent.

Before we can compute the homology of the homotopy limit $\|\mathcal{D}\|$ of a $(P, G)$ diagram $\mathcal{D}$ we have to introduce some facts about Whitney homology. The Whitney homology of a simplicial complex $\Delta$ is the homology of $\Delta$ with respect to the differential $\partial$ which maps an $n$-simplex $\left(x_{0}, \ldots, x_{n}\right)$ to $\sum_{i=0}^{n-1}(-1)^{i}\left(x_{0}, \ldots, \hat{x_{i}}, \ldots, x_{n}\right)$. Whitney homology of order complexes of posets is studied in [Ba] and [Bj2]. For a finite group $G$ and a $\mathbb{Z}[G]$-module $M$, we denote by $W H_{i}(\Delta, M)$ the $i$ th Whitney homology of $\Delta$ with coefficients in $M$. We shall use the following result of Björner [Bj2]. Björner's original formulation does not involve group actions, but the same proof gives the following proposition. (See [Su, Theorem 1.2].)

Proposition 2.2 (Theorem 5.1, [Bj2]). Let $P$ be a G-poset for a finite group $G$ and let $M$ be a $\mathbb{Z}[G]$-module. Assume that $\hat{0}$ is the least and $\hat{1}$ is the greatest element of $P$, and assume that $P / G$ is a set of representatives of $G$-orbits on $P$. Then the following isomorphism of $G$-modules holds :

$$
W H_{i}(\Delta(P), M) \cong \bigoplus_{x \in P / G} \operatorname{Ind}_{G_{x}}^{G} \widetilde{H}_{i}(\Delta([\hat{0}, x]), M) .
$$

Proposition 2.3. Let $\mathcal{D}$ be a $(P, G)$-diagram satisfying (HOM). The following isomorphism of graded $G$-modules holds :

$$
\widetilde{H}_{*}(\|\mathcal{D}\|) \cong_{G} \bigoplus_{x \in P / G} \operatorname{Ind}_{G_{x}}^{G}\left(\widetilde{H}_{*-1}\left(\Delta\left(\widehat{P_{<x}}\right) \otimes \widetilde{H}_{*}\left(\mathcal{D}_{x}\right)\right) .\right.
$$

Proof. We denote by $\hat{0}$ an artificial least element of $P$. The proof is based on the argument given in [Se, (5.1)]. For an $n \geq 0$ and a poset $Q$ we denote by $\Delta(Q)^{n}$ the set of all $i$-simplices in $\Delta(Q)$ for $i \leq n$ (i.e. the $n$-skeleton of $\Delta(Q))$. By " $\equiv_{n}$ " we denote the restriction of "=" to the $G$-space $\|\mathcal{D}\|^{n}:=\coprod_{x \in P} \Delta\left(\widehat{P_{\leq x}}\right)^{n} \times \mathcal{D}_{x}$. In 
particular this gives rise to a filtration of $G$-spaces

$$
\|\mathcal{D}\|^{0}=\left(\coprod_{x \in P} \Delta\left(\widehat{P_{\leq x}}\right)^{0} \times \mathcal{D}_{x} / \equiv_{0}\right) \hookrightarrow\|\mathcal{D}\|^{1} \hookrightarrow \cdots \hookrightarrow\|\mathcal{D}\|=\coprod_{x \in P} \Delta\left(\widehat{P_{\leq x}}\right) \times \mathcal{D}_{x} / \equiv
$$

Assume that the product $\left(x_{0}<x_{1}<\cdots<x_{n}\right) \times c$ is an $(n+m)$-cell in the space $\coprod_{x_{0}<\cdots<x_{n}}\left(x_{0}<\cdots<x_{n}\right) \times \mathcal{D}_{x_{n}}$. The differential $\partial\left(\left(x_{0}<x_{1}<\cdots<x_{n}\right) \times c\right)$ of the corresponding cell in $\|\mathcal{D}\|$ is then given by

$$
\begin{aligned}
& \sum_{i=1}^{n-1}(-1)^{i}\left(x_{0}<\cdots<\hat{x}_{i}<\cdots<x_{n}\right) \times c \\
&+(-1)^{n}\left(x_{0}<\cdots<x_{n-1}\right) \times d_{x_{n} x_{n-1}}(c) \\
&+(-1)^{n+1}\left(x_{0}<\cdots<x_{n}\right) \times \partial(c)
\end{aligned}
$$

In the spectral sequence associated to the filtration $\|\mathcal{D}\|^{n}$ the $E^{1}$-term is given by $E_{n, m}^{1}=H_{n+m}\left(\|\mathcal{D}\|^{n},\|\mathcal{D}\|^{n-1}\right)$. From the construction of $\|\mathcal{D}\|$ we infer that there is as a $G$-module $E_{n, m}^{1}$ is isomorphic to

$$
\bigoplus_{\sigma \in \Delta(P)^{n} / G} \operatorname{Ind}_{S t a b(\sigma)}^{G} H_{m}\left(D_{\max (\sigma)}\right),
$$

where $\max (\sigma)$ denotes the maximal element of the $n$-simplex $\sigma$ and $\Delta(P)^{n} / G$ denotes a set of representatives of orbits of $n$-simplicies.

Thus the differential $d^{1}$ of the $E^{1}$ term in our spectral sequence is given by the first two terms in (2.1), By (HOM) the second term vanishes, thus only the first term contributes to the $E_{n, m}^{2}$. But this implies that $D^{1}$ is the differential of the Whitney homology with coefficients in the $S \operatorname{tab} \max (\sigma)$-modules $H_{m}\left(D_{\max (\sigma)}\right.$. Therefore by Proposition $2.2 E_{n, m}^{2}$ is given by the following formula.

$$
E_{n, m}^{2}=\bigoplus_{x \in P / G} \operatorname{Ind}_{G_{x}}^{G} H_{n-1}\left(\Delta(\hat{0}, x), \widetilde{H}_{m}\left(\mathcal{D}_{x}\right)\right)
$$

It is easily seen from (2.1) that the differential $d^{2}$ is 0 in this case (see also [Se] for discussions of the general case). Moreover, since we are working with homology with coefficients in the rationals, we can apply the Universal Coefficient Theorem. Therefore the homology of $\|\mathcal{D}\|$ is given by

$$
\widetilde{H}_{i}(\|\mathcal{D}\|) \cong_{G} \bigoplus_{x \in P / G} \bigoplus_{i=n+m} \operatorname{Ind}_{G_{x}}^{G}\left(\widetilde{H}_{n-1}(\Delta(\hat{0}, x)) \otimes \widetilde{H}_{m-1}\left(\mathcal{D}_{x}\right)\right) .
$$

Note that we use only the fact that our coefficients are in a field of characteristic 0 in the last step. The same argument goes through if we assume that the integral cohomology of $\mathcal{D}_{x}$ is free for all $x \in P$. In particular this is true for diagrams of spaces induced by arrangements of linear subspaces. But we shall not use this fact except in the Theorem 2.4 (i), since we do not pursue representation theory over $\mathbb{Z}$.

Theorem 2.4. (i) [Go-MacP], [Hu], [Je], [Va2], [Zie-Ž] Let $\mathcal{A}$ be an arrangement of linear subspaces in $\mathbb{R}^{n}$. Then

$$
\widetilde{H}_{i}\left(D_{\mathcal{A}}\right) \cong \bigoplus_{x \in L_{\mathcal{A}}^{>0}} \widetilde{H}_{i-\operatorname{dim}(x)-1}([\hat{0}, x], \mathbb{Z})
$$


(ii) Let $\mathcal{A}$ be a $G$-arrangement of linear subspaces in $\mathbb{R}^{n}$ for a finite subgroup $G$ of $G l_{n}(\mathbb{R})$. Then the following isomorphism of $G$-modules holds

$$
\widetilde{H}_{i}\left(D_{\mathcal{A}}\right) \cong_{G} \bigoplus_{x \in\left(L_{\mathcal{A}}^{>0}\right) / G} \operatorname{Ind}_{G_{x}}^{G}\left(\widetilde{H}_{i-\operatorname{dim}(x)-1}([\hat{0}, x]) \otimes \widetilde{H}_{\operatorname{dim}(x)-1}\left(S_{x}^{(\operatorname{dim}(x)-1)}\right)\right) .
$$

Proof. The intersection of a linear subspace of $\mathbb{R}^{n}$ of dimension $k$ with a the unit sphere $S^{n-1}$ is homeomorphic to a $(k-1)$-sphere $S^{k-1}$. Now let $W<V$ be two subspaces in the arrangement $\mathcal{A}$. Then $V \cap S^{n-1}$ is a $S^{\operatorname{dim}(V)-1}$-sphere embedded in the $(\operatorname{dim}(W)-1)$-sphere $W \cap S^{n-1}$. These embeddings induce a trivial map

$$
\widetilde{H}_{*}\left(\bigcup_{W<V} V \cap S^{n-1}\right) \rightarrow \widetilde{H}_{*}\left(W \cap S^{n-1}\right) .
$$

Therefore the arrangement $\mathcal{A}$ satisfies (HOM), and the assertion now follows from Proposition 2.3 (for part (i) use the remark preceding this theorem).

By Alexander duality, taking orientation into account, we deduce the following equivariant Goresky-MacPherson formula (part (ii) below). For a subspace $x$ of $\mathbb{R}^{n}$ we denote by $x^{\perp}$ its orthogonal complement with respect to the $G$-invariant symmetric form $\langle,\rangle_{G}$.

Theorem 2.5. (i) [Go-MacP], [Hu], [Je], [Va2], [Zie-Ž] Let $\mathcal{A}$ be an arrangement of linear subspaces in $\mathbb{R}^{n}$. Then

$$
\widetilde{H}^{i}\left(\mathcal{M}_{\mathcal{A}}\right) \cong \bigoplus_{x \in L_{\mathcal{A}}^{>\hat{0}}} \widetilde{H}_{\operatorname{codim}(x)-i-2}(\Delta([\hat{0}, x])) .
$$

(ii) Let $\mathcal{A}$ be a $G$-arrangement of linear subspaces in $\mathbb{R}^{n}$ for a finite subgroup $G$ of $G l_{n}(\mathbb{R})$. Then the following isomorphism of $G$-modules holds

$$
\widetilde{H}^{i}\left(\mathcal{M}_{\mathcal{A}}\right) \cong_{G} \bigoplus_{x \in\left(L_{\mathcal{A}}^{>\hat{0}}\right) / G} \operatorname{Ind}_{G_{x}}^{G}\left(\widetilde{H}_{\operatorname{codim}(x)-i-2}(\Delta([\hat{0}, x])) \otimes \widetilde{H}_{\operatorname{codim}(x)-1}\left(S_{x^{\perp}}^{\operatorname{codim}(x)-1}\right)\right) .
$$

Proof. Let $\pi$ denote the mapping which assigns to each $x \in \mathcal{M}_{\mathcal{A}}$ the closest intersection of the line through $x$ and the origin with the unit sphere $S^{n-1}$. Then $\pi$ is easily seen to be a deformation retract which is $G$-equivariant. The image of $\mathcal{M}_{\mathcal{A}}$ under $\pi$ is the complement of the link $D_{\mathcal{A}}$ in $S^{n-1}$. In particular $\widetilde{H}^{i}\left(\mathcal{M}_{\mathcal{A}}\right) \cong \widetilde{H}^{i}\left(S^{n-1}-D_{\mathcal{A}}\right)$ is an isomorphism of $G$-modules.

Now part (i) follows immediately by Alexander duality [Mu, 71.1] on the sphere. For part (ii) we have to look at the Alexander duality isomorphism a bit more carefully. The Alexander duality isomorphism for the subset $D_{\mathcal{A}}$ of $S^{n-1}$ is given (see proof of [Mu, 71.1]), for $i>0$, by

$$
\widetilde{H}^{i}\left(S^{n-1}-D_{\mathcal{A}}\right) \cong H_{n-1-i}\left(S^{n-1}, D_{\mathcal{A}}\right) \cong \widetilde{H}_{n-1-i}\left(D_{\mathcal{A}}\right) .
$$

The first isomorphism is induced by Lefschetz duality and the second isomorphism is given by the mapping $\partial^{*}$, induced by the boundary operator $\partial$ of the pair $\left(S^{n-1}, D_{\mathcal{A}}\right)$. Moreover $\partial^{*}$ is a $G$-equivariant mapping, whereas Lefschetz duality commutes with the group action only modulo orientation. For $i=0$, a modified argument yields the same final result: the Alexander duality isomorphism commutes with the group action up to orientation.

In precise terms, the effect of the group action is the following, for arbitrary $i \geq 0$. Let $o$ be an arbitrary orientation of the $G$-invariant sphere $S^{n-1}$. Then $o$ determines $([\mathrm{Mu}, \S 72])$ a Lefschetz duality mapping $\gamma_{o}$. For an arbitrary cocycle $c \in \widetilde{H}^{i}\left(\mathcal{M}_{\mathcal{A}}\right)$ 
and an element $g \in G l_{n}(\mathbb{R})$ we have $\left(\gamma_{o}(c)\right)^{g}=\gamma_{o^{g}}\left(c^{g}\right)$. In particular the mapping is $G$-equivariant up to orientation: $o^{g}=\operatorname{det}(g) \cdot o$. Hence $\left(\gamma_{o}(c)\right)^{g}=\operatorname{det}(g) \cdot \gamma_{o}\left(c^{g}\right)$. Since $\operatorname{det}(g)$ is the character of $g$ on the homology of the sphere $S^{n-1}$, Theorem 2.4 implies that $\widetilde{H}^{i}\left(\mathcal{M}_{\mathcal{A}}\right)$ is isomorphic to $\widetilde{H}_{n-1-i}\left(D_{\mathcal{A}}\right) \otimes \widetilde{H}_{n-1}\left(S^{n-1}\right)$ as a $G$-module.

For any $x \in \mathcal{A}$ the sphere $S^{n-1}$ is $G_{x}$-homeomorphic to the join

$$
S_{x}^{\operatorname{dim}(x)-1} * S_{x^{\perp}}^{\operatorname{codim}(x)-1}
$$

Therefore $\widetilde{H}_{n-1}\left(S^{n-1}\right) \cong \widetilde{H}_{\operatorname{dim}(x)-1}\left(S_{x}^{\operatorname{dim}(x)-1}\right) \otimes \widetilde{H}_{\operatorname{codim}(x)-1}\left(S_{x^{\perp}}^{\operatorname{codim}(x)-1}\right)$ as $G_{x^{-}}$ modules. By basic manipulations the assertion now follows from Theorem 2.4.

Example 2.6. (i) Let $\mathcal{A}$ consist of a single hyperplane $H$ in $\mathbb{R}^{n}$. Then $\mathcal{A}$ is invariant under the cyclic group $G_{1}$ of order 2 generated by the reflection in $H$. $\mathcal{A}$ is also invariant under the cyclic group $G_{2}$ generated by a reflection along a hyperplane containing $H^{\perp}$. (Note that $L_{\mathcal{A}}$ contains only a single point.) Now $G_{1}$ (resp. $G_{2}$ ) preserves the orientation on $S^{n-1} \cap H$ (resp. $S^{n-1} \cap H^{\perp}$ ) and reverses the orientation on $S^{n-1} \cap H^{\perp}$ (resp. $\left.S^{n-1} \cap H\right)$. Hence from the proof of the previous theorem we deduce that $G_{1}$ (resp. $G_{2}$ ) acts trivially (resp. by mutiplication by -1 ) on $\widetilde{H}_{n-2}\left(D_{\mathcal{A}}\right)$ and by multiplication by -1 (resp. trivially) on $\widetilde{H}_{0}\left(\mathcal{M}_{\mathcal{A}}\right)$.

(ii) Let $\mathcal{A}$ be an arrangement of hyperplanes in $\mathbb{R}^{n}$. Let $G$ be a finite subgroup of $G l_{n}(\mathbb{R})$ that acts faithfully as a group of permutations on the set of connected components of $\mathcal{M}_{\mathcal{A}}$. Then Theorem 2.5 provides a decomposition of the permutation module $H^{0}\left(\mathcal{M}_{\mathcal{A}}\right)$ - which, in some cases (see Corollary 4.5) turns out to be a new occurrence of a classical one.

(iii) Assume the situation of (ii). Let $\mathcal{A}^{\mathbb{C}}$ be the complexification of the arrangement $\mathcal{A}$ (i.e., the complex hyperplanes in $\mathbb{C}^{n}$ defined by the same forms as the hyperplanes in $\mathbb{R}^{n}$ in $\mathcal{A}$ ). Then the $G$-modules $H^{i}\left(\mathcal{M}_{\mathcal{A}^{\mathbb{C}}}\right)$ are essentially the same as those occurring in the decomposition of $H^{0}\left(\mathcal{M}_{\mathcal{A}}\right)$ but the different twists by modules of the form $\widetilde{H}_{\operatorname{codim}(x)-1}\left(S_{x^{\perp}}^{\operatorname{codim}(x)-1}\right)$ produce an interesting vanishing property of the alternating sum (see Corollary 2.11).

In order to determine the representation of $G$ on $\widetilde{H}^{i}\left(\mathcal{M}_{\mathcal{A}}\right)$ we have to understand the representations of finite subgroups $G$ of $G l_{n}(\mathbb{R})$ on the homology $\widetilde{H}_{n-1}\left(S^{n-1}\right)$. The following elementary fact will give the key information which is necessary in this context.

Lemma 2.7. (i) Let $G$ be a finite subgroup of $G l_{n}(\mathbb{R})$. Let $d>1$ be an integer. Let $G(d) \cong G$ be the diagonal of the subgroup $\underbrace{G \times \cdots \times G}_{d \text { times }}$ of $G l_{n d}(\mathbb{R})$. Then $G$ acts in an orientation-preserving manner on $S^{d n-1}$ if either $d$ is even or if $G$ acts in an orientation-preserving manner on $S^{n-1}$.

(ii) Let $G$ be a finite subgroup of $G l_{n}(\mathbb{C})$. Then $G$ acts in an orientationpreserving manner on $S^{2 n-1}$.

Proof. The first assertion follows from the fact that the action of a matrix $A$ preserves the orientation on the unit sphere if and only if $\operatorname{det}(A)=1$. By the same argument it suffices to show for the second assertion, that if we regard $G$ as a subgroup of $G l_{2 n}(\mathbb{R})$, then all elements of $G$ have determinant +1 . Now an arbitrary matrix of finite order in $G L_{n}(\mathbb{C})$ has determinant $\prod_{j=1}^{n} e^{\varphi_{j} i}$ (as usual $e$ denotes the 
base of the natural logarithm). Each $e^{\varphi_{j} i}$ acts on $\mathbb{C} \cong \mathbb{R}^{2}$ by rotations. In particular its determinant as an element of $G l_{2}(\mathbb{R})$ is 1 .

A good example of the difference between subgroups of $G L_{n}(\mathbb{C})$ and $G l_{n}(\mathbb{R})$ in the preceding lemma is given by the action of complex conjugation on $\mathbb{C}^{n}$. If $G \cong C_{2}$ is the cyclic group of order two generated by the complex conjugation, its action on $\widetilde{H}_{2 n-1}\left(S^{2 n-1}\right)$ is nontrivial.

The next corollary generalises an analogous result for complex hyperplane arrangements of Orlik and Solomon [O-So, Corollary 4.7]. In particular the following corollary applies for complexified arrangements. For an arrangement of subspaces $\mathcal{A}$ in $\mathbb{R}^{n}$ we denote by $\mathcal{A}^{\mathbb{C}}$ the arrangement of linear subspaces in $\mathbb{C}^{n}$ which are defined by the same (real) equations as the subspaces in $\mathcal{A}$. In particular $L_{\mathcal{A}}$ and $L_{\mathcal{A}^{\mathbb{C}}}$ are isomorphic lattices. The arrangement $\mathcal{A}^{\mathbb{C}}$ is called the complexified arrangement corresponding to the arrangement $\mathcal{A}$. If $\mathcal{A}$ is a $G$-arrangement for the group $G \leq G l_{n}(\mathbb{R})$ then $\mathcal{A}^{\mathbb{C}}$ is a $G$-arrangement for the group $G$ considered as a subgroup of $G l_{n}(\mathbb{C})$ (see also Lemma 2.7 (i)), with the action on a complex vector $\underline{\mathbf{x}}+i \underline{\mathbf{y}}$ given by

$$
A(\underline{\mathbf{x}}+i \underline{\mathbf{y}})=A \underline{\mathbf{x}}+i A \underline{\mathbf{y}}
$$

for all $A \in G$.

Corollary 2.8. Let $\mathcal{A}$ be a $G$-arrangement of $\mathbb{C}$-linear subspaces in $\mathbb{C}^{n}$ for a finite subgroup $G$ of $G l_{n}(\mathbb{C})$. Then the following isomorphism of $G$-modules holds

$$
\widetilde{H}^{i}\left(\mathcal{M}_{\mathcal{A}}\right) \cong_{G} \bigoplus_{x^{G} \in\left(L_{\mathcal{A}}^{>\hat{0}}\right) / G} \operatorname{Ind}_{G_{x}}^{G}\left(\widetilde{H}_{\operatorname{codim}(x)-i-2}([\hat{0}, x])\right) .
$$

Before proceeding to the next corollary we recall a well-known theorem (see for example [Bre, p. 120]).

Proposition 2.9. Let $K$ be a simplicial complex on which a finite group $G$ acts as a group of simplicial automorphisms. Let $\widetilde{H}_{i}(\cdot)$ denote reduced singular homology with coefficients in a field of characteristic 0 . Then for all $i$ there is an ismorphism of the space of $G$-invariants $\widetilde{H}_{i}(|K|)^{G}$ of the $G$-module $\widetilde{H}_{i}(|K|)$ and the homology $\widetilde{H}_{i}(|K| / G)$.

Note that the dimension of the space $\widetilde{H}_{i}(|K|)^{G}$ is given by the multiplicity of the trivial $G$-representation on $\widetilde{H}_{i}(|K|)$. Of course the analogous statement holds for singular cohomology. As an immediate corollary of Theorem 2.4 and Proposition 2.9 we obtain:

Corollary 2.10. Let $\mathcal{A}$ be a $G$-arrangement of $\mathbb{C}$-linear subspaces in $\mathbb{C}^{n}$ for a finite subgroup $G$ of $G l_{n}(\mathbb{C})$. Then

$$
\widetilde{H}^{i}\left(\mathcal{M}_{\mathcal{A}} / G\right) \cong \widetilde{H}_{n-i-1}\left(D_{\mathcal{A}} / G\right) .
$$

For example, if $G=S_{n}$ acts by permuting coordinates on a $G$-invariant arrangement $\mathcal{A}$, then Corollary 2.10 holds by Alexander duality even for integral cohomology. In this case the one-point compactification $S^{2 n}$ of $\mathbb{C}^{n}$ is $G$-homeomorphic (the point at infinity is a fixed point) to the suspension of $S^{2 n-1}$. The sphere $S^{2 n}$ can be decomposed as the disjoint union of the suspension $\Sigma D_{\mathcal{A}}$ of $D_{\mathcal{A}}$ and $\mathcal{M}_{\mathcal{A}}$. Since $\mathbb{C}^{n} / S_{n} \cong \mathbb{C}^{n}$, we deduce that $S^{2 n} \cong S^{2 n} / S_{n} S^{n} \cong \Sigma\left(D_{\mathcal{A}} / S_{n}\right) \cup\left(\mathcal{M}_{\mathcal{A}} / S_{n}\right)$. 
This proves the assertion via Alexander duality on the sphere. But it remains open whether in general the duality of Corollary 2.10 holds for integral coefficients.

The following corollary generalises results given by Orlik and Solomon [O-So] for complex hyperplane arrangements.

Corollary 2.11. Let $\mathcal{A}$ be a $G$-arrangement of linear subspaces in $\mathbb{R}^{n}$ for a finite subgroup $G$ of $G l_{n}(\mathbb{R})$. Then the virtual $G$-module

$$
\sum_{i \geq 0}(-1)^{i} H^{i}\left(\mathcal{M}_{\mathcal{A}^{\mathbb{C}}}\right)
$$

vanishes. Moreover the Euler characteristic of $\mathcal{M}_{\mathcal{A}} / G$ is 0 .

Proof. The first assertion follows from the "equivariant acyclicity" of the Whitney homology established in [Su, Lemma 1.1]. This means that the virtual $G$-module

$$
\sum_{i \geq 0}(-1)^{i} W H_{i}(P)
$$

vanishes for an arbitrary poset $P$. The second assertion follows from the first by Proposition 2.9 .

\section{The group action on the Whitney homology of the lattice $\Pi_{\lambda}$,}

$$
\lambda=\left(k, 1^{n-k}\right) \text { AND } \lambda=\left(k^{\ell}\right) .
$$

This section serves to recall the results and notation from $[\mathrm{Su}-\mathrm{Wa}]$ and $[\mathrm{Su}]$. In Section 4 these results will be applied to the study of the orbit and configuration spaces associated to the manifolds $\mathcal{M}_{\lambda}^{d}$. We shall make use of the representationtheoretic results in [Su-Wa] for $\lambda=\left(k, 1^{n-k}\right)$, and those in [Su] for $\lambda=\left(k^{\ell}\right)$.

Let $\mu=\left(\mu_{1} \geq \mu_{2} \geq \ldots\right)$ be an integer partition of $n$. Denote by $S_{\mu}$ the Young subgroup of $S_{n}$ corresponding to the partition $\mu$, i.e., $S_{\mu}$ is isomorphic to $\underset{i \geq 1}{\times} S_{\mu_{i}}$.

We denote by $S_{n}[G]$ the wreath product of the symmetric group $S_{n}$ with the group $G$. Finally we write $N_{S_{n}}\left(S_{\mu}\right)$ for the normaliser of the Young subgroup $S_{\mu}$ in $S_{n}$. Using the notation $\mu=\left(1^{m_{1}}, 2^{m_{2}}, \ldots\right)$ to indicate that $m_{j}$ is the multiplicity of the part $j$ in the integer partition $\mu$, then clearly $N_{S_{n}}\left(S_{\mu}\right)$ is the direct product of wreath products $\underset{j}{\times} S_{m_{j}}\left[S_{j}\right]$.

In the sequel we will need much of the notation of [Su, Section 1]. For background on symmetric functions and plethysm, we refer to [Macd]. As in [Macd] we denote the Frobenius characteristic by ch. Let $G$ be a finite group. If $V$ is an $S_{m}$-module and $W$ is an $G$-module, we write $V[W]$ for the wreath product module canonically induced by the representations $\otimes^{m} W$ of $\times^{m} G$ and $V$ of $S_{m}$.

When $G$ is itself a symmetric group $S_{c}$, we write $\operatorname{ch}(V[W])$ for the characteristic of the $S_{m c}$-character of the induced module $\operatorname{Ind}_{S_{m}\left[S_{c}\right]}^{S_{m c}}(V[W])$. We shall need the following fact about wreath product modules: the characteristic $\operatorname{ch}(V[W])$ is the plethysm $\operatorname{ch}(V)[\operatorname{ch}(W)]$.

For an arbitrary partition $\lambda$ of $n$, the Schur function indexed by $\lambda$ is denoted $s_{\lambda}$ as in [Macd]. We shall use the notation $s_{\lambda}$ for both the corresponding irreducible representation and its Frobenius characteristic (i.e., the Schur function for this shape). In particular for $n \geq k \geq 2$, we denote by $s_{\left(k, 1^{n-k}\right)}$ the irreducible representation of $S_{n}$ indexed by the hook shape $\left(k, 1^{n-k}\right)$.

We write $h_{n}$ for the trivial representation $s_{(n)}$ and $e_{n}$ for the sign representation $s_{\left(1^{n}\right)}$. (In the notation of [Macd], $h_{n}$ is the homogeneous symmetric function and 
$e_{n}$ is the elementary symmetric function.) Finally as in [Macd] we denote by $\omega$ the involution in the ring of symmetric functions which takes $h_{n}$ to $e_{n}$.

Two particular symmetric functions will arise frequently in our calculations in Section 4 . These are $\pi_{n}$, the Frobenius characteristic of the $S_{n}$-action on the homology $\widetilde{H}_{n-3}\left(\Pi_{n}\right)$ of the full partition lattice $\Pi_{n}$, and $\ell_{n}$, the characteristic of the $S_{n}$-action on the multilinear component, of total degree $n$, of the free Lie algebra. It is a well known fact that $\ell_{n}=\omega\left(\pi_{n}\right)$; in terms of $S_{n}$-actions this says that the two representations differ only up to tensoring by the sign.

Recall from Section 2, (or [Bj2, Theorem 5.1]), that the Whitney homology in degree $p \geq 0$ of a poset $P$, is given by

$$
W H_{p}(P)=\bigoplus_{x \in P} \widetilde{H}_{p-2}(\hat{0}, x)
$$

Theorem 3.1 ([Su-Wa, Theorem 3.7]). The contribution in degree $p>0$ of the representation on the Whitney cohomology of $\Pi_{\left(k, 1^{n-k}\right)}$, tensored with the sign, is given by the degree $n$ term in the characteristic

$$
\sum_{s \geq 0}\left(\left.\sum_{t \geq 1} \omega^{k}\left(\sum_{r \geq 1} e_{r}\left[\sum_{i \geq 1} \ell_{i}\right]\right)\right|_{\operatorname{deg} t}\right)\left[\sum_{j \geq k} s_{\left(j-k+1,1^{k-1}\right)}\right] e_{s},
$$

where the sums range over all subscripts $r, s$ and $t$ such that $p=n-r-s-t(k-2)$. Note that $W H_{0}\left(\Pi_{\left(k, 1^{n-k}\right)}\right)$ is the trivial $S_{n}$-module.

Finally we record the same calculations for the lattice $\Pi_{\left(k^{\ell}\right)}$ consisting of partitions in $\Pi_{k \ell}$ all of whose block sizes are divisible by $k$. The representation of the symmetric group on the top homology of $\Pi_{\left(k^{\ell}\right)}$ was first determined in [C-H-R]. For the purposes of the computations in the next section, we shall need the representation on the Whitney homology as described in $[\mathrm{Su}]$. Denote the characteristic of the action of $S_{k l}$ on the (top) homology $\widetilde{H}_{\ell-2}\left(\Pi_{\left(k^{\ell}\right)}\right)$ by $\pi_{\left(k^{\ell}\right)}^{\ell-2}$. We have

Theorem 3.2 ([Su, Lemma 1.1 and Theorem 5.3]). The characteristics $\pi_{\left(k^{\ell}\right)}^{\ell-2}$ are determined recursively by

$$
\pi_{\left(k^{\ell}\right)}^{\ell-2}=\sum_{i=0}^{\ell-1}(-1)^{\ell-1-i} \operatorname{ch}\left(W H_{r}\left(\Pi_{\left(k^{\ell}\right)}\right)\right) .
$$

The characteristic of the rth Whitney homology of $\Pi_{\left(k^{\ell}\right)}$, for $r=1, \ldots, n$, is given by

$$
\operatorname{ch}\left(W H_{r}\left(\Pi_{\left(k^{\ell}\right)}\right)\right)=\sum_{\substack{\lambda \vdash k \ell, \ell(\lambda)=\ell+1-r \\ \lambda=\left(k^{m_{1}},(2 k)^{m_{2}}, \ldots\right)}} \prod_{i \geq 1} h_{m_{2 i-1}}\left[\pi_{\left(k^{2 i-1}\right)}^{2 i-3}\right] e_{m_{2 i}}\left[\pi_{\left(k^{2 i}\right)}^{2 i-2}\right] .
$$

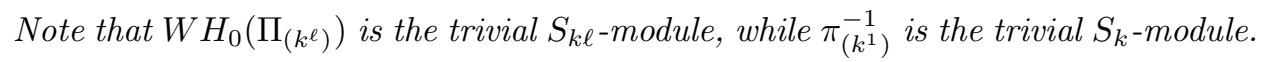

\section{ORdered AND UNORDERED CONFIGURATION SPACES}

In this section we use the results of Section 3 to analyse the topology and (co)homology of the orbits of the $G$-operation on the link $\mathcal{D}_{\mathcal{A}}$ and on the complement $\mathcal{M}_{\mathcal{A}}$ of the $G$-arrangements $\mathcal{A}$.

We shall state some general results on the ordered configuration spaces $\mathcal{M}_{\lambda}^{d}$ and the unordered configuration spaces $\mathcal{M}_{\lambda}^{d} / S_{n}$, for an integer partition $\lambda$ of $n$. In order 
to do this, we first record, in Example 4.1 below, the sign twists which arise in the Goresky-MacPherson formula Theorem 2.4 (ii). These are the representations on the homology of the spheres $S_{x}^{\operatorname{dim}(x)-1}$ and $S_{x^{\perp}}^{\operatorname{codim}(x)-1}$ in Theorem 2.4 (ii) and Theorem 2.5 (ii), which are induced by the action of the stabiliser of a subspace on the homology of its intersection with the unit sphere $S^{n-1}$. The statements in Example 4.1 follow from elementary linear algebra; the proofs are therefore omitted.

Example 4.1. (i) Let $\tau$ be a reflection along a hyperplane in $\mathbb{R}^{n}$. Then $\tau$ acts by multiplication with -1 on $\widetilde{H}_{n-1}\left(S^{n-1}\right)$. This follows from the fact that the reflection $\tau$ has determinant -1 and hence acts on $S^{n-1}$ in an orientation-reversing manner.

(ii) Let $\lambda=\left(1^{m_{1}}, 2^{m_{2}}, \ldots, n^{m_{n}}\right)$ be an integer partition of $n$. Let $G \cong S_{n}$ be the the group of monomial matrices in $G l_{n}(\mathbb{R})$ with entries 0,1 . Then the action of the normaliser $N_{S_{n}}\left(S_{\lambda}\right)=S_{m_{1}}\left[S_{1}\right] \times S_{m_{2}}\left[S_{2}\right] \times \cdots \times S_{m_{n}}\left[S_{n}\right]$ of $S_{\lambda}$ in $S_{n}$ on $\widetilde{H}_{n-1}\left(S^{n-1}\right)$ is given by the restriction $\bigotimes_{i \geq 1} e_{m_{2 i-1}}\left[e_{2 i-1}\right] \otimes h_{m_{2 i}}\left[e_{2 i}\right]$ of the sign representation of $S_{n}$ to $N_{S_{n}}\left(S_{\lambda}\right)$.

(iii) Let $\mathcal{A}_{\lambda}^{d}$ be the orbit arrangement with parameter $\lambda \vdash n$ in $\mathbb{R}^{d n}$ for an ODD number $d$. Let $x \in L_{\mathcal{A}_{\lambda}^{d}}=\Pi_{\lambda}$ be the subspace corresponding to the partition $\mu=\left(1^{m_{1}}, 2^{m_{2}}, \ldots, n^{m_{n}}\right)$. Then the normaliser of $S_{\mu}$ in $S_{n}$ acts on $S_{x}^{\operatorname{dim}(x)-1}$ by $\bigotimes_{i \geq 1} e_{m_{i}}\left[h_{i}\right]$ and on $S_{x^{\perp}}^{\operatorname{codim}(x)-1}$ by $\bigotimes_{i \geq 1} h_{m_{2 i-1}}\left[e_{2 i-1}\right] \otimes e_{m_{2 i}}\left[e_{2 i}\right]$. Since the sphere $S^{n-1}$ is homeomorphic to the join of spheres $S_{x}^{\operatorname{dim}(x)-1} * S_{x^{\perp}}^{\operatorname{codim}(x)-1}$, the Künneth formula implies that the representation of $S_{n}$ on the sphere $S^{n-1}$ is given by the tensor product of the two preceding representations. This confirms the result of part (i).

Proposition 4.2. Let $\lambda$ be an integer partition of $n$.

(i) Let $d=1$. The orbit space $\mathcal{M}_{\lambda}^{1} / S_{n}$ is contractible for all partitions $\lambda$ of $n$.

(ii) If $d$ is an arbitrary odd number, then $\widetilde{H}^{i}\left(\mathcal{M}_{\lambda}^{d} / S_{n}\right)=0$ for all $i$.

(iii) Let $l(\lambda)$ denote the length of the partition $\lambda$. If $d$ is an arbitrary even number, then $\widetilde{H}^{i}\left(\mathcal{M}_{\lambda}^{d} / S_{n}\right) \cong \mathbb{Q}$ for $i=d \cdot(n-l(\lambda))-1$. Moreover for any $d \geq 1$, the cohomology of $\mathcal{M}_{\lambda}^{d}$, and hence of its orbit space, vanishes in degrees less than $d \cdot(n-l(\lambda))-1$.

(iv) $\chi\left(\mathcal{M}_{\lambda}^{d} / S_{n}\right)=0$ if $d$ is even and $\chi\left(\mathcal{M}_{\lambda}^{d} / S_{n}\right)=1$ if $d$ is odd.

Proof. (i) The orbit space $\mathbb{R}^{n} / S_{n}$ is homeomorphic to a product of a copy of the real line with a closed $(n-2)$-dimensional simplicial cone. Geometrically it can be identified with the subset of $\mathbb{R}^{n}$ consisting of points $\left(x_{1}, \ldots, x_{n}\right)$ such that $x_{1} \leq \cdots \leq x_{n}$. Now the orbit of $S^{n-1}$ under the $S_{n}$ action corresponds to the points $\left(x_{1} \leq \cdots \leq x_{n}\right)$ at unit distance from the origin. In particular the orbit of the link $D_{\mathcal{A}_{(2,1 n-2)}^{1}}$ is homeomorphic to the $S^{n-2}$-sphere in the boundary of the orbit space $S^{n-1} / S_{n}$. The interior of $S^{n-1} / S_{n}$ is homeomorphic to an $(n-1)$-ball. It follows that the orbit of $\mathcal{M}_{\left(2,1^{n-2}\right)}^{1}$ is homeomorphic to the direct product of the real line with a cone over an open $(n-1)$-ball. In particular it is contractible. Now the orbit space $\mathcal{M}_{\lambda}^{1} / S_{n}$ is the space $\mathcal{M}_{\left(2,1^{n-2}\right)}^{1} / S_{n}$, with some cells added to the boundary. But adding cells to the boundary preserves the property of being contractible. 
(ii) By part (i) the space $\mathcal{M}_{\lambda}^{1} / S_{n}$ is contractible. This in turn implies by Proposition 2.9 that the trivial representation does not occur in $\bigoplus_{i>0} \widetilde{H}_{i}\left(\mathcal{M}_{\lambda}^{1}\right)$. By the Goresky-MacPherson formula, Theorem 2.4 (ii), and by Example 4.1 (iii), the sum $\bigoplus_{i \geq 0} \widetilde{H}_{i}\left(\mathcal{M}_{\lambda}^{1}\right)$ and the sum $\bigoplus_{i \geq 0} \widetilde{H}_{i}\left(\mathcal{M}_{\lambda}^{d}\right)$ are isomorphic $G$-modules for odd $d$. Consequently the trivial representation does not occur in the latter sum for any odd $d$.

(iii) First we claim that for arbitrary $d \geq 1$, the cohomology of $\mathcal{M}_{\lambda}^{d}$ vanishes if $i<$ $d(n-l(\lambda))-1$. From the ordinary Goresky-MacPherson formula, the contribution to cohomology in degree $i$ is $\bigoplus_{x \in \Pi_{\lambda}} \widetilde{H}_{j(x, i)}(\hat{0}, x)$, where $j(x, i)=\operatorname{codim}(x)-i-2$. If $x$ is a partition of type $\mu$, then $\operatorname{codim}(x)=d(n-l(\mu))$. The length of the longest chain from $\hat{0}$ to $x$ is bounded above by $l(\lambda)-l(\mu)+1$, and hence the homology of the interval $(\hat{0}, x)$ in $\Pi_{\lambda}$ occurs in degree equal to at most $l(\lambda)-l(\mu)-1$. This gives $j(x, i)=d(n-l(\mu))-i-2 \leq l(\lambda)-l(\mu)-1$, or equivalently, $i \geq$ $(d-1)(n-l(\mu))+(n-l(\lambda))-1$. The claim follows by observing that the minimum value of $i$ is achieved for the maximum value of $l(\mu)$, which occurs when $\mu=\lambda$.

In particular it follows that if $d \geq 2$, only elements of type $\lambda$ contribute to the lowest degree in the cohomology. Hence for $d \geq 2, \widetilde{H}^{d(n-l(\lambda))-1}\left(\mathcal{M}_{\lambda}^{d}\right)$ is free with rank equal to the number of atoms of $\Pi_{\lambda}$.

Now we examine the representation on the cohomology in this dimension. For a partition $x$ of type $\lambda$ the homology $\widetilde{H}_{*}([\hat{0}, x])$ is concentrated in dimension -1 , and affords the trivial $N_{S_{n}}\left(S_{\lambda}\right)$ action. Therefore $\operatorname{Ind}_{N_{S_{n}}\left(S_{\lambda}\right)}^{S_{n}} \widetilde{H}_{*}([\hat{0}, x])$ is a transitive permutation module. Theorem 2.4 (ii) and the remarks preceding this paragraph imply that for even $d, \widetilde{H}^{d(n-l(\lambda))-1}\left(\mathcal{M}_{\lambda}^{d}\right)$ contains the trivial representation exactly once. The statement about the orbit space now follows from Proposition 2.9.

(iv) For odd $d$ this follows from part (ii). For even $d$ the assertion is implied by the second part of Corollary 2.11 for $d=2$. For arbitrary even $d$ the claim follows from Theorem 2.4 (ii) and Lemma 2.7 (ii). They show that the trivial representation is shifted by an even degree when passing from $d=2$ to another even $d \geq 2$.

In particular this implies the following result about spaces of polynomials with restricted root multiplicities.

Corollary 4.3. Let $\lambda$ be an integer partition of $n$. Then the space $\mathcal{M}_{\lambda}^{2} / S_{n}$ of monic polynomials in $\mathbb{C}[x]$ of degree $n$ with multiplicity of zeros $\mu$ such that $\mu$ is not coarser than $\lambda$ satisfies:

(i) $\widetilde{H}^{2 n-2 \cdot l(\lambda)-1}\left(\mathcal{M}_{\lambda}^{2} / S_{n}\right) \cong \mathbb{Q}$.

(ii) $\widetilde{H}^{i}\left(\mathcal{M}_{\lambda}^{2} / S_{n}\right)=0$ for $i<2 n-2 \cdot l(\lambda)-1$.

Proof. This follows directly from Proposition 4.2 by the usual identification of $\mathbb{C}^{n} / S_{n} \cong \mathbb{C}^{n}$ with the space of monic polynomials of degree $n$ in $\mathbb{C}[x]$.

Although for odd $d$ the orbit space $\mathcal{M}_{\lambda}^{d} / S_{n}$ is acyclic over the rational numbers, it is not true that in general $\mathcal{M}_{\lambda}^{d} / S_{n}$ is contractible. This is of course a wellknown fact, but we include the argument for the sake of completeness. Consider for arbitrary $d$ the map that sends an element $\underline{\mathbf{x}}=\left(x_{1}, \ldots, x_{n}\right)$ to its "average" 
$f(\underline{\mathbf{x}})=\frac{1}{n} \sum_{i=1}^{n} x_{i} \in \mathbb{R}^{d}$. This map induces a trivial $S_{n}$-invariant fiber bundle of $\mathcal{M}_{\lambda}^{d}$ over $\mathbb{R}^{d}$, whose fiber is the complement of the arrangement $\mathcal{A}_{\lambda}^{d}$ in the subspace $V_{\underline{\mathbf{0}}} \cong \mathbb{R}^{(n-1) d}$, defined by $\frac{1}{n} \sum_{i=1}^{n} x_{i}=\underline{\mathbf{0}}$. Since $f$ is $S_{n}$-equivariant over the trivial $S_{n}$-space $\mathbb{R}^{d}$ this induces a trivial fiber bundle of the orbit space $\mathcal{M}_{\lambda}^{d} / S_{n}$ over $\mathbb{R}^{d}$. Its fiber is the orbit of $V_{\underline{0}}-\mathcal{A}_{\lambda}^{d}$ under the action of $S_{n}$. Now assume $\lambda=\left(2,1^{n-2}\right)$ and $n=2$. Thus $V_{\underline{\mathbf{0}}}=\left\{(x,-x) \mid x \in \mathbb{R}^{d}\right\}$ and $V_{\underline{\mathbf{0}}}-\mathcal{A}_{\lambda}^{d}=\{(x,-x) \mid \underline{\mathbf{0}} \neq x \in$ $\left.\mathbb{R}^{d}\right\} \cong \mathbb{R}^{d}-\underline{\mathbf{0}}$. The $S_{2}$ action corresponds to the reflection $x \mapsto-x$ on $\mathbb{R}^{d}$. In particular $\left\{(x,-x) \mid \underline{\mathbf{0}} \neq x \in \mathbb{R}^{d}\right\} / S_{2}$ is homotopic to real projective $(d-1)$-space. Therefore it is not contractible for $d>1$. More precisely, for $d>1$ we have,

$$
\widetilde{H}^{i}\left(\mathcal{M}_{(2)}^{d} / S_{2}, \mathbb{Z}\right)= \begin{cases}\mathbb{Z}_{2} & \text { if } i \text { is odd and } 0<i<d-1, \\ \mathbb{Z} & \text { if } d-1 \text { is odd and } i=d-1, \\ 0 & \text { otherwise }\end{cases}
$$

Now we begin investigating properties of the spaces $\mathcal{M}_{\left(k, 1^{n-k}\right)}^{d}$. Before we study representations and orbit spaces, we shall give a description of the integral cohomology of $\mathcal{M}_{\left(k, 1^{n-k}\right)}^{d}$. For $d=1$ and $d=2$ this has been done in [Bj-We] and the general statement is a simple corollary of their methods. By the results of Björner and Welker, it is clear that $\widetilde{H}_{i}\left(\Pi_{\left(k, 1^{n-k}\right)}, \mathbb{Z}\right)$ is a torsion-free $\mathbb{Z}$-module and is nontrivial if and only if $i=n-3-t(k-2)$ for some $1 \leq t \leq\left\lfloor\frac{n}{k}\right\rfloor$. We shall denote by $B_{\left(k, 1^{n-k}\right)}^{i}$ the $i$ th Betti number of the complex $\Delta\left(\Pi_{\left(k, 1^{n-k}\right)}\right)$. Recall also from Section 3 that $\ell_{n}$ denotes (the characteristic of) the $S_{n}$-representation on the multilinear part of the free Lie algebra on $n$ generators, and that $\omega\left(\ell_{n}\right)=\pi_{n}$, where $\pi_{n}$ is the representation on the top homology of $\Pi_{n}$.

Theorem 4.4. (i) [Bj-We, Theorem 5.2, Theorem 5.4] The integral cohomology of $\mathcal{M}_{\left(k, 1^{n-k}\right)}^{d}$ is free and is nontrivial in dimension $i$ if and only if

$$
t \cdot(k-2)+(d-1)(t k-m) \leq i \leq t(k-2)+(d-1)(n-m)
$$

for some $1 \leq m \leq t \leq\left\lfloor\frac{n}{k}\right\rfloor$.

(ii) [Bj-We, Theorem 5.2, Theorem 5.4] If $t \cdot(k-2)+(d-1)(t k-m) \leq i \leq$ $t(k-2)+(d-1)(n-m)$ for some $1 \leq m \leq t \leq\left\lfloor\frac{n}{k}\right\rfloor$, the ith Betti number of $\mathcal{M}_{\left(k, 1^{n-k}\right)}^{d}$ is given by the sum

$$
\sum_{1 \leq m \leq t \leq\left\lfloor\frac{n}{k}\right\rfloor} \sum_{\substack{a_{1}+\cdots+a_{m} \leq n \\ a_{i} \geq k}} \sum_{\substack{t_{1}+\cdots+t_{m}=t \\ 1 \leq t_{j} \leq\left\lfloor\frac{a_{j}}{k}\right\rfloor}} B_{\left(k, 1^{a_{1}-k}\right)}^{a_{1}-t_{1}(k-2)-3} \cdots B_{\left(k, 1^{a_{m}-k}\right)}^{a_{m}-t_{m}(k-2)-3} .
$$

(iii) Write $H_{k}$ for the sum of symmetric functions $\sum_{j \geq k} s_{\left(j-k+1,1^{k-1}\right)}$. The characteristic of the $S_{n}$-representation on the $i$-dimensional cohomology of the manifold $\mathcal{M}_{\left(k, 1^{n-k}\right)}^{d}$, equals the degree $n$ term in the sum

$$
\sum_{r, t \geq 1, s \geq 0 i=(d-1)(n-r-s)+t(k-2)} \psi_{r, s, t}
$$


where $\psi_{r, s, t}$ is given by

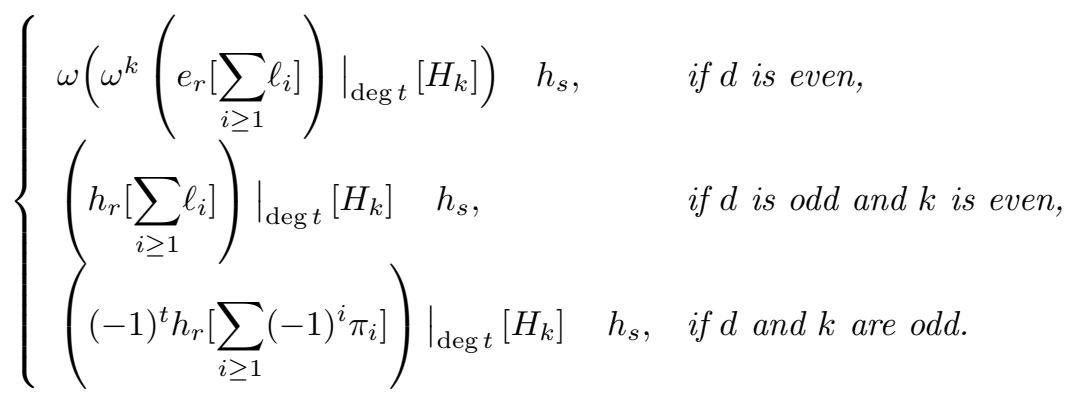

When $k=2$, these formulas simplify, so that the $S_{n}$-module structure of the cohomology group $\widetilde{H}^{i}\left(\mathcal{M}_{\left(2,1^{n-2}\right)}^{d}\right)$, for $i=(d-1)(n-p), 1 \leq p \leq n-1$, is given by the degree $n$ term in

$$
\begin{cases}\omega\left(e_{p}\left[\sum_{i \geq 1} \ell_{i}\right]\right), & \text { if } d \text { is even } \\ h_{p}\left[\sum_{i \geq 1} \ell_{i}\right], & \text { if } d \text { is odd }\end{cases}
$$

Proof. The proof of parts (i) and (ii) follows essentially the lines of the proof given in [Bj-We, Theorems 5.2, Theorem 5.4] for $d=1,2$. In order to get contribution to cohomology in dimension $i$, by the Goresky-MacPherson formula we must have that $\widetilde{H}_{\operatorname{codim}(x)-i-2}([\hat{0}, x])$ does not vanish for some $x \in \Pi_{\left(k, 1^{n-k}\right)}$. Now let $a_{1}, \ldots, a_{m}$ be the sizes of the blocks in $x \in \Pi_{\left(k, 1^{n-k}\right)}$ which are nontrivial (i.e. have size $\geq k$ ), $s=\left|\left\{a_{i} \mid a_{i}=1\right\}\right|$ and $m_{j}=\left|\left\{a_{i} \mid a_{i}=j\right\}\right|$ for $j \geq k$. By [Bj-We, Theorem 1.5] the homology of the posets $\Pi_{\left(k, 1^{n-k}\right)}$ is free. The interval $[\hat{0}, x]$ is isomorphic to the direct product $\underset{j=k}{\times}\left(\Pi_{\left(k, 1^{j-k}\right)}\right)^{m_{j}}$. By the Künneth formula its homology is also free and from [Bj-We, Theorem 4.8], is concentrated in dimensions

$$
\begin{gathered}
a_{1}-t_{1}(k-2)-3+\cdots+a_{m}-t_{m}(k-2)-3+2(m-1) \\
=\sum_{j=1}^{m}\left(a_{i}-t_{i}(k-2)\right)-m-2,
\end{gathered}
$$

for some $1 \leq t_{i} \leq\left\lfloor\frac{a_{i}}{k}\right\rfloor$. The codimension of the subspace corresponding to the partition $x$ is $(n-m-s) d$. We set $t=\sum_{i=1}^{m} t_{i}$. Obviously $n \geq t \geq m$. We use the observation $s=n-\sum_{j=1}^{m} a_{j}$. By the Goresky MacPherson formulas, Theorem 2.5 (i) and (ii), the interval $[\hat{0}, x]$ contributes to $\widetilde{H}^{*}\left(\mathcal{M}_{\left(k, 1^{n-k}\right)}^{d}, \mathbb{Z}\right)$ in dimension $i=t(k-2)+\left(\sum_{j=1}^{m} a_{j}-m\right)(d-1)$. Now assertion (i) follows by the fact that $t k \leq \sum_{j=1}^{m} a_{j} \leq n$ and all integer values in this range are assumed.

This implies assertions (i) and (ii) via the Goresky-MacPherson formulas, Theorem 2.5 (i). 
For assertion (iii), we use the computation in [Su-Wa] of the Whitney homology module of $\Pi_{\left(k, 1^{n-k}\right)}$, as recorded in Section 3. The case of even $d$ is essentially the formula for the Whitney homology of $\Pi_{\left(k, 1^{n-k}\right)}$ (tensored with the sign) from Theorem 3.1, while the case of odd $d$ comes from using Example 4.1 (iii) in conjunction with this formula. In both cases we use the fact that if $x$ is a partition in $\Pi_{\left(k, 1^{n-k}\right)}$ with $r$ nontrivial blocks and $s$ singletons, then the codimension function is given by $\operatorname{codim}(x)=d(n-r-s)$.

We now describe the details of the computation for odd $d$. The notation is as in the proof of [Su-Wa, Theorem 3.7]. Recall that the contribution from the interval $[\hat{0}, x]$ to the representation on $W H_{j}\left(\Pi_{\left(k, 1^{n-k}\right)}\right)$ factors into

$$
\bigoplus_{\nu} \operatorname{Ind}_{N_{S_{n}}\left(S_{\nu}\right)}^{S_{n}}\left(B^{\nu} \otimes P^{\nu}\right)
$$

where the integer partition $\nu$ of $n$ is the type of $x$, with $s$ parts equal to 1 and $n-s$ parts all greater than or equal to $k$, such that $j=n-s-r+t(k-2)$.

From the Goresky-MacPherson formula for $G$-modules, Theorem 2.5 (ii), the representation on the cohomology of the complement of the real manifold is obtained by tensoring each factor in the above with the action of the normaliser $N_{S_{n}}\left(S_{\nu}\right)$ on the homology of the sphere $S_{x^{\perp}}^{\operatorname{codim}(x)-1}$, where $x \in \Pi_{\left(k, 1^{n-k}\right)}$ is of type $\nu$. The action on the sphere $S_{x}^{\operatorname{dim}(x)-1}$ is trivial for the Young subgroup $S_{\nu}$, while, by Example 4.1 (iii), the outer factors of the normaliser act like the sign. Hence the $N_{S_{n}}\left(S_{\nu}\right)$-module $\widetilde{H}\left(S_{x}^{\operatorname{dim}(x)-1}\right)$ has characteristic $\chi_{n, r, s}$, where, in the notation of [Su, Remark 1.4.0],

$$
\chi_{n, r, s}=\left(\operatorname{Res}_{\times_{j} S_{m_{j}}} e_{r}\right)\left[\bigotimes_{j \geq k} h_{j}\right] \otimes e_{s} ;
$$

here $m_{j}$ is the multiplicity of the part $j \geq k$ in $\nu$, i.e., the number of blocks of size $j \geq k$ in $x$. By Example 4.1 (iii) the action on $\widetilde{H}\left(S_{x^{\perp}}^{\operatorname{codim}(x)-1}\right)$ is given by tensoring the representation $\chi_{n, r, s}$ with the sign.

The contribution to the cohomology of the complement $\mathcal{M}_{\left(k, 1^{n-k}\right)}^{d}$ from elements $x$ of type $\nu$ is thus

$$
\operatorname{Ind}_{N_{S_{n}}\left(S_{\nu}\right)}^{S_{n}}\left(B^{\nu} \otimes P^{\nu} \otimes \operatorname{sgn} \otimes\left(\chi_{n, r, s}\right)\right)=\operatorname{Ind}_{N\left(S_{\nu}\right)}^{S_{n}}\left(\operatorname{sgn} \otimes\left(B^{\nu}\right) \otimes P^{\nu} \otimes \chi_{n, r, s}\right) .
$$

To simplify the calculations, write $\phi_{n, r, s, t}$ for the term of degree $n-s$ in

$$
\left.\omega^{k}\left(e_{r}\left[\sum_{i \geq 1} \ell_{i}\right]\right)\right|_{\operatorname{deg} t}\left[\sum_{j \geq k} s_{\left(j-k+1,1^{k-1}\right)}\right] .
$$

Then, as in the proof of [Su-Wa, Theorem 3.7], the contribution to the cohomology of the complement from the Whitney homology of degree $j$ is

$$
\begin{gathered}
\omega\left(\operatorname{ch}\left(W H_{j}\left(\Pi_{\left(k, 1^{n-k}\right)}\right)\right)\right) \otimes e_{r}\left[\sum_{j \geq k} h_{j}\right] e_{s} \\
=\sum_{s \geq 0} h_{s}\left(\sum_{r, t \geq 1} \phi_{n, r, s, t} \otimes \chi_{n, r, s}\right)
\end{gathered}
$$

where the sum ranges over all subscripts $r, s$ and $t$ such that $j-2=n-r-s-$ $t(k-2)-2$. 
When $k$ is even, the result is clear.

When $k$ is odd, the computation of the tensor product $\phi_{n, r, s, t} \otimes \chi_{n, r, s}$ is more intricate. Note first that by the rule of signs for plethysms of homogeneous symmetric functions, we can write

$$
\left.\omega\left(e_{r}\left[\sum_{i \geq 1} \ell_{i}\right]\right)\right|_{\operatorname{deg} t}=\left.(-1)^{t} e_{r}\left[\sum_{i \geq 1}(-1)^{i} \pi_{i}\right]\right|_{\operatorname{deg} t} .
$$

Hence for odd $k$, we find that $\phi_{n, r, s, t} \otimes \chi_{n, r, s}$ is the term of degree $(n-s)$ in

$$
\left.\left((-1)^{t} h_{r}\left[\sum_{i \geq 1}(-1)^{i} \pi_{i}\right]\right)\right|_{\operatorname{deg} t}\left[\sum_{j \geq k} s_{\left(j-k+1,1^{k-1}\right)}\right],
$$

as in the statement of the theorem.

We would like to mention that Theorem 4.4 (i) implies that $\widetilde{H}_{i}\left(\mathcal{M}_{\left(k, 1^{q}\right)}, \mathbb{Z}\right)$ vanishes for $i>\frac{n}{k}(k-2)+(d-1)(n-1)$ (this result is due to Cohen and Lusk $[\mathrm{Co}-\mathrm{Lu}])$. We begin our analysis with an observation on the spaces $D_{\mathcal{A}_{\left(2,1^{n-2}\right)}^{1}}$ and $\mathcal{M}_{\left(2,1^{n-2}\right)}^{1}$, which are of lesser interest topologically. Here Theorems 2.4 (ii) and 2.5 (ii) allow us to retrieve a known decomposition of the regular representation of $S_{n}$. Part (i) of the following corollary is of course well-known and stated for the sake of completeness.

Corollary 4.5. (i) The action of $S_{n}$ on $H^{0}\left(\mathcal{M}_{\mathcal{A}_{\left(2,1^{n-2}\right)}}\right)$ is the regular representation.

(ii) The $S_{n}$-representation on $\widetilde{H}^{0}\left(\mathcal{M}_{\mathcal{A}_{\left(2,1^{n-2}\right)}}\right)$ is given by

$$
\sum_{\substack{m_{1}, m_{2}, \ldots, m_{n} \geq 0 \\ \sum_{i} i m_{i}=n, \sum_{i} m_{i} \leq n-1}} \prod_{i \geq 1} h_{m_{i}}\left[\ell_{i}\right] .
$$

Note that, together with part (i), this is equivalent to an $S_{n}$-equivariant formulation of the Poincaré-Birkhoff-Witt theorem.

(iii) The regular representation of $S_{n}$ decomposes into a direct sum of $\bigoplus_{i \geq 0} \widetilde{H}_{i}\left(D_{\mathcal{A}_{\left(2,1^{n-2}\right)}}\right)$ and a copy of the sign representation. The $S_{n}$-representation on $\bigoplus_{i \geq 0} \widetilde{H}_{i}\left(D_{\left.\mathcal{A}_{\left(2,1^{n-2}\right)}\right)}\right)$ is given by

$$
\sum_{\substack{m_{1}, m_{2}, \ldots, m_{n} \geq 0 \\ \sum_{i} i m_{i}=n, \sum_{i} m_{i} \leq n-1}} \prod_{i \geq 1} e_{m_{2 i-1}}\left[\pi_{2 i-1}\right] h_{m_{2 i}}\left[\pi_{2 i}\right] .
$$

Proof. The hyperplanes in the arrangement $\mathcal{A}_{\left(2,1^{n-2}\right)}^{1}$ dissect $\mathbb{R}^{n}$ into $n$ ! pieces, each homeomorphic to a simplicial cone times a copy of the real line. The symmetric group $S_{n}$ acts transitively on the $n$ ! pieces. Therefore $S_{n}$ acts on $H^{0}\left(\mathcal{M}_{\left(2,1^{n-2}\right)}^{1}\right)$ by the regular representation. Now assertion (ii) follows by using the GoreskyMacPherson formula for $G$-modules, and Example 4.1 (iii). Note that the contribution to the Whitney homology of $\Pi_{\left(2,1^{n-2}\right)}$, as an $S_{n}$-module, from set partitions $x$ of type $\lambda=\left(1^{m_{1}}, 2^{m_{2}}, \ldots\right)$ is given by $\prod_{i \geq 1} h_{m_{2 i-1}}\left[\pi_{2 i-1}\right] e_{m_{2 i}}\left[\pi_{2 i}\right]$. 
Part (iii) can be deduced similarly, using the Goresky-MacPherson formula Theorem 2.5 (ii).

Here we remind the reader of the fact that the two representations in Corollary 4.5 arise naturally in the given decomposition from each other via "equivariant" Alexander duality on the sphere (see the proof of Theorem 2.4) and also via the involution $\omega$. The action of $\omega$ can therefore be interpreted in this context as topological duality on the sphere.

In order to apply Proposition 2.9 to the manifolds $\mathcal{M}_{\left(k, 1^{n-k}\right)}^{d}$ we have to determine the multiplicity of the trivial representation in the $S_{n}$-module $\widetilde{H}^{*}\left(\mathcal{M}_{\left(k, 1^{n-k}\right)}^{d}\right)$. The key ingredient here is the computation in $[\mathrm{Su}-\mathrm{Wa}]$ of the representation on the Whitney homology of $\Pi_{\left(k, 1^{n-k}\right)}$, which is stated in Theorem 3.1.

Proposition 4.6. Let $d$ be even. The multiplicity of the trivial representation in the reduced cohomology of the complement of $\mathcal{M}_{\left(k, 1^{n-k}\right)}^{d}$, is 1 in degree $d(k-1)-1$, and zero elsewhere. In particular

$$
\widetilde{H}^{i}\left(\mathcal{M}_{\left(k, 1^{n-k}\right)}^{d} / S_{n}\right)= \begin{cases}\mathbb{Q} & \text { if } i=d(k-1)-1, \\ 0 & \text { otherwise. }\end{cases}
$$

Proof. By Proposition 2.9 and Theorem 4.4 (iii), it is enough to show that the trivial representation occurs in the Whitney homology of $\Pi_{\left(k, 1^{n-k}\right)}$ only in dimension 0 and 1.

Clearly $W H_{0}\left(\Pi_{\left(k, 1^{n-k}\right)}\right)$ is the trivial module. Now let $l>0$.

We compute the multiplicity of the sign representation in the tensor product of the sign with $W H_{p}\left(\Pi_{\left(k, 1^{n-k}\right)}\right)$, using Frobenius reciprocity. The scalar product of the symmetric function $e_{n}$ with the expression given by Theorem 3.1 is equal to the sum over all $s \geq 0$ of the scalar product of $e_{n-s}$ with

$$
\left(\left.\sum_{t=r}^{\left\lfloor\frac{n-s}{k}\right\rfloor} \omega^{k}\left(\sum_{r \geq 1} e_{r}\left[\sum_{i \geq 1} \ell_{i}\right]\right)\right|_{\operatorname{deg} t}\right)\left[e_{k}\right] .
$$

This in turn is nonzero only for those $t$ such that $n-s=k t$, in which case, using the fact that the restriction of the sign representation from $S_{k t}$ to $S_{t}\left[S_{k}\right]$ is given by $h_{t}\left[e_{k}\right]$ if $k$ is even, and by $e_{t}\left[e_{k}\right]$ if $k$ is odd, we find that the multiplicity equals the scalar product of $\omega^{k} h_{t}$ with the degree $t$ term in

$$
\omega^{k}\left(\sum_{r \geq 1} e_{r}\left[\sum_{i \geq 1} \ell_{i}\right]\right) .
$$

Finally this is the multiplicity of the trivial representation in the degree $t$ term of

$$
\sum_{r \geq 1} e_{r}\left[\sum_{i \geq 1} \ell_{i}\right]
$$

It is well-known that the trivial representation appears in $\ell_{i}$ if and only if $i=1$. (Equivalently, the sign representation appears in $\pi_{i}$ if and only if $i=1$. This follows easily, for instance, from [St2, Theorem 7.3].) Hence the multiplicity of the trivial representation in $W H_{p}\left(\Pi_{\left(k, 1^{n-k}\right)}\right)$ is nonzero if and only if

$$
r=t=1 \quad \text { and } \quad n-s=k,
$$

that is, if and only if $p=n-s-r-t(k-2)=1$, in which case it is 1 . 
Another example of an orbit arrangement is the $k$-divisible arrangement $\mathcal{A}_{\left(k^{\ell}\right)}^{d}$ in $\mathbb{R}^{k \cdot \ell \cdot d}$, whose intersection lattice $\Pi_{\left(k^{\ell}\right)}$ (usually denoted by $\Pi_{k}^{\ell}$ ) is the join sublattice of $\Pi_{k \ell}$, consisting of partitions all of whose block sizes are divisible by $k$ (see [Bj4, Example 3.3]). Note that $\Pi_{\left(k^{\ell}\right)}$ is a ranked lattice of rank $\ell$. We denote the $i$ th Betti-numbers of $\widetilde{H}_{*}\left(\Pi_{\left(k^{\ell}\right)}\right)$ by $B_{\left(k^{\ell}\right)}^{i}$.

Proposition 4.7. (i) [Wa] For arbitrary $k$ and $\ell$ the poset $\Pi_{\left(k^{\ell}\right)}$ is EL-shellable. All its homology is concentrated in degree $\ell-2$.

(ii) [St1] The dimension $B_{\left(k^{\ell}\right)}^{\ell-2}$ of $\widetilde{H}_{\ell-2}\left(\Pi_{\left(k^{\ell}\right)}\right)$ is equal to the number of permutations in $S_{\ell k-1}$ with descent set $\{k, 2 k, \ldots,(\ell-1) k\}$.

The representation $\pi_{\left(k^{\ell}\right)}^{\ell-2}$ of the symmetric group on the top homology of $\Pi_{\left(k^{\ell}\right)}$ was first studied in $[\mathrm{C}-\mathrm{H}-\mathrm{R}]$. Shellability was first proved by Wachs and later independently by Sagan [Sa]. The representation on the Whitney homology of this lattice is recorded in Theorem 3.2. From this we derive the representation of $S_{k \ell}$ on the cohomology of the complement of the $\ell$-divisible arrangement, via the GoreskyMacPherson formula Theorem 2.5. As in the $k$-equal case, we can also extract information about the orbit space.

Theorem 4.8. (i) [Bj4, Example 3.3] The integral cohomology of $\mathcal{M}_{\left(k^{\ell}\right)}^{d}$ is free and is nontrivial in dimension $i$ if and only if $i=\ell(d k-1)-t(d-1)-1$ for some $1 \leq t \leq n$.

(ii) If $i=\ell(d k-1)-t(d-1)-1$ for some $1 \leq t \leq \ell$ and $d>1$, then the $i$ th Betti number of $\mathcal{M}_{\left(k^{\ell}\right)}^{d}$ is given by the sum

$$
\sum_{\substack{\lambda \vdash k \ell, \ell(\lambda)=t \\ \lambda=\left(k^{m_{1}},(2 k)^{m_{2}}, \ldots\right)}}(\underbrace{k \cdots k}_{m_{1}} \cdots \underbrace{k \ell}_{m_{\ell}} \cdot k k \ell) \cdot m_{1} ! \cdots m_{\ell} ! \cdot B_{(k)}^{m_{1}} \cdots B_{\left(k^{\ell}\right)}^{m_{\ell}} .
$$

If $d=1$ then the $\widetilde{H}^{i}\left(\mathcal{M}_{\left(k^{\ell}\right)}^{d}\right)$ is nontrivial if and only if $i=\ell(d k-1)-1$, and this Betti number is given by

$$
\sum_{t=1}^{\ell} \sum_{\substack{\lambda \vdash k \ell, \ell(\lambda)=t \\ \lambda=\left(k^{m_{1}},(2 k)^{m_{2}}, \ldots\right)}}(\underbrace{k \cdots k}_{m_{1}} \cdots \underbrace{k \ell}_{m_{\ell}} \cdots k \ell) \cdot m_{1} ! \cdots m_{\ell} ! \cdot B_{(k)}^{m_{1}} \cdots B_{\left(k^{\ell}\right)}^{m_{\ell}} .
$$

(iii) Let $i=\ell(d k-1)-t(d-1)-1$ for some $1 \leq t \leq n$ and $d>1$. The characteristic of the $S_{k \ell-m o d u l e ~} \widetilde{H}^{i}\left(\mathcal{M}_{\left(k^{\ell}\right)}^{d}\right)$ is

$$
\sum_{\substack{\lambda+k \ell, \ell(\lambda)=t \\ \lambda=\left(k^{m},(2 k)^{m_{2}}, \ldots\right)}} \chi_{\lambda}
$$

where

$$
\chi_{\lambda}= \begin{cases}\prod_{i \geq 1} h_{m_{2 i-1}}\left[\pi_{\left(k^{2 i-1}\right)}^{2 i-3}\right] e_{m_{2 i}}\left[\pi_{\left(k^{2 i}\right)}^{2 i-2}\right], & \text { if } d \text { is even, } \\ \prod_{i \geq 1} e_{m_{2 i-1}}\left[\omega \pi_{\left(k^{2 i-1}\right)}^{2 i-3}\right] h_{m_{2 i}}\left[\omega \pi_{\left(k^{2 i}\right)}^{2 i-2}\right], & \text { if } d \text { is odd and } k \text { even } \\ \prod_{i \geq 1} h_{m_{i}}\left[\omega \pi_{\left(k^{i}\right)}^{i-2}\right] & \text { if } d \text { and } k \text { are odd }\end{cases}
$$


In particular when $d$ and $k$ are both odd, it follows from (2) that the $S_{k \ell}$-module structure of the cohomology of the complement is that of a symmetric algebra: its characteristic is given by the degree $n$ term in

$$
\sum_{t=1}^{\ell} h_{t}\left[\sum_{i \geq 1} \omega \pi_{\left(k^{i}\right)}^{i-2}\right]
$$

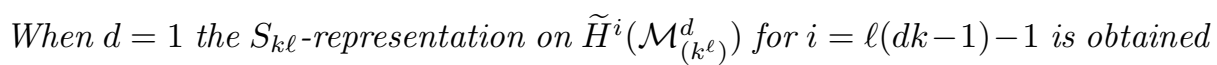
by taking the sum over all $1 \leq t \leq \ell$ in the expressions (1) and (2) above.

Proof. By Proposition 4.7 (i) the integral homology of $\Pi_{\left(k^{\ell}\right)}$ is free and concentrated in dimension $\operatorname{rank}\left(\Pi_{\left(k^{\ell}\right)}\right)-1=\ell-2$. A partition $x$ in $\Pi_{\left(k^{\ell}\right)}$ with $t$ blocks corresponds to a subspace of codimension $d \ell k-d t$. If $x$ is a partition of type $\left(k^{m_{1}}, \ldots,(\ell k)^{m_{\ell}}\right)$ then the lower interval $[\hat{0}, x]$ in $\Pi_{\left(k^{\ell}\right)}$ is isomorphic to a direct product $\underset{i=1}{\stackrel{\ell}{\times}}\left(\Pi_{\left(k^{i}\right)}\right)^{m_{i}}$. Of course $t=\sum_{i=1}^{\ell} m_{i}$ and $\sum_{i=1}^{\ell} m_{i} i=\ell$. The homology of the (Cohen-Macaulay) interval $[\hat{0}, x]$ is concentrated in the top dimension $\ell-t-1$. Therefore the interval $[\hat{0}, x]$ contributes to $\widetilde{H}^{*}\left(\mathcal{M}_{\left(k^{\ell}\right)}^{d}, \mathbb{Z}\right)$ in dimensions

$$
i=d \ell k-d t-\ell-t-1=\ell(d k-1)-t(d-1)-1 .
$$

Now the assertions (i)-(iii) follow from the Goresky-MacPherson formulas in Theorem 2.5, Example 4.1 (iii), and Theorem 3.2.

Proposition 4.9. For an even number $d$, the multiplicity of the trivial representation in the reduced cohomology of $\mathcal{M}_{\left(k^{\ell}\right)}^{d}$, is 1 in degree $(\ell d-1)(k-1)-1$, and zero elsewhere. In particular

$$
\widetilde{H}^{i}\left(\mathcal{M}_{\left(k^{\ell}\right)}^{d} / S_{n}\right)= \begin{cases}\mathbb{Q}, & \text { if } i=(\ell d-1)(k-1)-1 \\ 0, & \text { otherwise. }\end{cases}
$$

Proof. Clearly the trivial representation appears in the homology of $\Pi_{\left(k^{\ell}\right)}$ once in the degenerate case $k=1$, and, for arbitrary $k$, exactly once in the Whitney homology of $\Pi_{\left(k^{\ell}\right)}$ in each of the degrees 0 and 1 . We prove by induction that it never appears in the homology of $\Pi_{\left(k^{\ell}\right)}$ for $k \geq 2$. From these facts the assertion then follows by Proposition 2.9 and Theorem 3.2.

The recursive formula in the preceding proposition for the characteristics $\pi_{\left(k^{\ell}\right)}$ follows from the equivariant acyclicity of Whitney homology established in [Su, Lemma 1.1]. For $k=2$ this gives $\pi_{\left(2^{\ell}\right)}^{\ell-2}=\operatorname{ch}\left(W H_{1}\left(\Pi_{\left(2^{\ell}\right)}\right)\right)-\operatorname{ch}\left(W H_{0}\left(\Pi_{\left(2^{\ell}\right)}\right)\right)$, and hence the trivial representation does not occur in $\pi_{\left(2^{\ell}\right)}^{\ell-2}$.

Assume by induction that it does not occur in $\pi_{\left(m^{\ell}\right)}^{\ell-2}$ for $2 \leq m \leq k-1$. Again by acyclicity

$$
\pi_{\left(k^{\ell}\right)}^{\ell-2}=\sum_{i=0}^{n-1}(-1)^{n-1-i} \operatorname{ch}\left(W H_{i}\left(\Pi_{\left(k^{\ell}\right)}\right)\right) .
$$

Examining the expression of Theorem 3.2 for the characteristic of the $r$ th Whitney homology, we find that there is a product of plethysms for each integer partition $\lambda$ of $k \ell$ with $k+1-r$ parts, with $m_{i}$ parts of size $i \ell$. Hence if $2 \leq r \leq k-1$, each such partition has at least one part of size greater than or equal to $2 k$. On the other hand all the part sizes are less than $k \ell$, so by induction hypothesis the trivial 
representation does not appear in $W H_{r}\left(\Pi_{\left(k^{\ell}\right)}\right)$ for $2 \leq r \leq k-1$. The contributions from degree 1 and 0 cancel each other, and it follows that the multiplicity of the trivial representation is 0 in $\pi_{\left(k^{\ell}\right)}^{\ell-2}$, for all $n \geq 2$.

It is now clear that the trivial module occurs once each in the Whitney homology in degrees 1 and 0 , and never elsewhere.

The results of Propositions 4.6 and 4.9 imply the following condition [Ar2] for $\lambda=\left(k, 1^{q}\right)$ and $\lambda=\left(k^{\ell}\right)$.

(Finiteness) Let $d$ be a positive even number. Then

$$
\widetilde{H}^{i}\left(\mathcal{M}_{\lambda}^{d} / S_{n}\right)= \begin{cases}\mathbb{Q} & \text { for } i=d n-d \cdot l(\lambda)-1, \\ 0 & \text { otherwise }\end{cases}
$$

The non-vanishing part of the (Finiteness) condition is precisely Proposition 4.2 (iii). Arnol'd introduces this condition in [Ar2], where he proves, for $d=2$ :

Proposition $4.10([\operatorname{Ar} 2])$. Let $\lambda=\left(k^{l}, 1^{q}\right)$ be a partition of $n=k . l+q$ for integers $k>1$ and $l \geq 1, q \geq 0$. Then the space $\mathcal{M}_{\lambda}^{2} / S_{n}$ satisfies the (Finiteness) condition.

Corollary 4.11. Let $\lambda=\left(k^{l}, 1^{q}\right)$ be a partition of $n=k . l+q$ for integers $k>1$ and $l \geq 1, q \geq 0$. Then for an even number $d$ the space $\mathcal{M}_{\lambda}^{d} / S_{n}$ satisfies the (Finiteness) condition.

Proof. By Theorem 2.5 and Lemma 2.7 the cohomology representations of $S_{n}$ on $\widetilde{H}^{*}\left(\mathcal{M}_{\lambda}^{d}\right)$ and $\widetilde{H}^{*}\left(\mathcal{M}_{\lambda}^{2}\right)$ differ only by dimension shifts. In particular the trivial representation occurs only once in $\widetilde{H}^{*}\left(\mathcal{M}_{\lambda}^{d}\right)$. Now the assertion follows from Proposition 4.2 (iii).

Based on Arnol'd's Theorem and some examples which we treated with the Goresky-MacPherson formula for $G$-modules (Theorem 2.5 (ii)), we conjecture the following generalisation of Arnol'd's Theorem:

Conjecture 4.12. For an arbitrary partition $\lambda$ of $n$, the space $\mathcal{M}_{\lambda}^{d} / S_{n}$ satisfies the (Finiteness) condition.

In view of Theorem 2.4 (ii) and Lemma 2.7, this is equivalent to the following more combinatorial statement.

Conjecture 4.13. Let $\lambda$ be an arbitrary partition of $n$. Let $x \in \Pi_{\lambda}$ be a partition of type $\mu \neq \lambda$. Then the multiplicity of the trivial representation in the $N_{S_{n}}\left(S_{\mu}\right)$ module $\widetilde{H}_{*}([\hat{0}, x])$ is 0 .

Finally we mention that since the formulation of Conjecture 4.13 does not involve the number $d$, it suffices to prove Conjecture 4.12 for a single (even) $d$.

\section{Character values; tables \& Results}

Table 1: Character values of $S_{3}$ on $\widetilde{H}^{i}\left(\mathcal{M}_{(3)}^{2}\right)$

$$
\begin{array}{|c||c|c|c|}
\hline \mathrm{i} \backslash \lambda & \left(1^{3}\right) & (2,1) & (3) \\
\hline 3 & 1 & 1 & 1 \\
\hline
\end{array}
$$

Table 2: Character values of $S_{4}$ on $\widetilde{H}^{i}\left(\mathcal{M}_{(3,1)}^{2}\right)$

\begin{tabular}{|c||c|c|c|c|c|}
\hline $\mathrm{i} \backslash \lambda$ & $\left(1^{4}\right)$ & $\left(2,1^{2}\right)$ & $\left(2^{2}\right)$ & $(3,1)$ & $(4)$ \\
\hline 3 & 4 & 2 & 0 & 1 & 0 \\
4 & 3 & 1 & -1 & 0 & -1 \\
\hline
\end{tabular}


Table 3: Character values of $S_{5}$ on $\widetilde{H}^{i}\left(\mathcal{M}_{\left(3,1^{2}\right)}^{2}\right)$

\begin{tabular}{|c||c|c|c|c|c|c|c|}
\hline $\mathrm{i} \backslash \lambda$ & $\left(1^{5}\right)$ & $\left(2,1^{3}\right)$ & $\left(2^{2}, 1\right)$ & $\left(3,1^{2}\right)$ & $(3,2)$ & $(4,1)$ & $(5)$ \\
\hline 3 & 10 & 4 & 2 & 1 & 1 & 0 & 0 \\
4 & 15 & 3 & -1 & 0 & 0 & -1 & 0 \\
5 & 6 & 0 & -2 & 0 & 0 & 0 & 1 \\
\hline
\end{tabular}

Table 4: Character values of $S_{6}$ on $\widetilde{H}^{i}\left(\mathcal{M}_{\left(3,1^{3}\right)}^{2}\right)$

\begin{tabular}{|c||c|c|c|c|c|c|c|c|c|c|c|}
\hline $\mathrm{i} \backslash \lambda$ & $\left(1^{6}\right)$ & $\left(2,1^{4}\right)$ & $\left(2^{2}, 1^{2}\right)$ & $\left(2^{3}\right)$ & $\left(3,1^{3}\right)$ & $(3,2,1)$ & $\left(3^{2}\right)$ & $\left(4,1^{2}\right)$ & $(4,2)$ & $(5,1)$ & $(6)$ \\
\hline 3 & 20 & 8 & 4 & 0 & 2 & 2 & 2 & 0 & 0 & 0 & 0 \\
4 & 45 & 9 & 1 & -3 & 0 & 0 & 0 & -1 & -1 & 0 & 0 \\
5 & 36 & 0 & -4 & 0 & 0 & 0 & 0 & 0 & 0 & 1 & 0 \\
6 & 20 & 2 & 0 & -2 & 2 & 2 & 2 & 0 & -2 & 0 & -2 \\
7 & 10 & 4 & 2 & -4 & 1 & 1 & 1 & 0 & -2 & 0 & -1 \\
\hline
\end{tabular}

Table 5 : Character values of $S_{7}$ on $\widetilde{H}^{i}\left(\mathcal{M}_{\left(3,1^{4}\right)}^{2}\right)$

\begin{tabular}{|c|c|c|c|c|c|c|c|c|}
\hline$i \backslash \lambda$ & $\left(1^{7}\right)$ & $\left(2,1^{5}\right)$ & $\left(2^{2}, 1^{3}\right)$ & $\left(2^{3}, 1\right)$ & $\left(3,1^{4}\right)$ & $\left(3,2,1^{2}\right)$ & \multicolumn{2}{|c|}{$\left(3,2^{2}\right)$} \\
\hline 3 & 35 & 15 & 7 & 3 & 5 & 3 & \multicolumn{2}{|c|}{1} \\
\hline 4 & 105 & 25 & 5 & -3 & 3 & 1 & \multicolumn{2}{|c|}{-1} \\
\hline 5 & 126 & 6 & -6 & -6 & 0 & 0 & \multicolumn{2}{|c|}{0} \\
\hline 6 & 140 & 10 & 0 & -2 & 8 & 4 & \multicolumn{2}{|c|}{0} \\
\hline 7 & 190 & 40 & 10 & -4 & 10 & 4 & & \\
\hline 8 & 105 & 25 & 5 & -3 & 3 & 1 & \multicolumn{2}{|c|}{-1} \\
\hline$i \backslash \lambda$ & $\left(3^{2}, 1\right)$ & 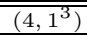 & $(4,2,1)$ & $(4,3)$ & $\overline{\left(5,1^{2}\right)}$ & $(5,2)$ & $(6,1)$ & $\overline{(7)}$ \\
\hline 3 & 2 & 1 & 1 & 1 & 0 & 0 & 0 & 0 \\
\hline 4 & 0 & -1 & -1 & -1 & 0 & 0 & 0 & 0 \\
\hline 5 & 0 & 0 & 0 & 0 & 1 & 1 & 0 & 0 \\
\hline 6 & 2 & 0 & -2 & 0 & 0 & 0 & -2 & 0 \\
\hline 7 & 1 & -2 & -4 & -2 & 0 & 0 & -1 & 1 \\
\hline 8 & 0 & -1 & -1 & -1 & 0 & 0 & 0 & 0 \\
\hline
\end{tabular}

Table 6: Character values of $S_{8}$ on $\widetilde{H}^{i}\left(\mathcal{M}_{\left(3,1^{5}\right)}^{2}\right)$

\begin{tabular}{|c||c|c|c|c|c|c|c|c|c|c|c|c|}
\hline $\mathrm{i} \backslash \lambda$ & $\left(1^{8}\right)$ & $\left(2,1^{6}\right)$ & $\left(2^{2}, 1^{4}\right)$ & $\left(2^{3}, 1^{2}\right)$ & $\left(2^{4}\right)$ & $\left(3,1^{5}\right)$ & $\left(3,2,1^{3}\right)$ & $\left(3,2^{2}, 1\right)$ & $\left(3^{2}, 1^{2}\right)$ & $\left(3^{2}, 2\right)$ & $\left(4,1^{4}\right)$ \\
\hline 3 & 56 & 26 & 12 & 6 & 0 & 11 & 5 & 3 & 2 & 2 & 4 \\
4 & 210 & 60 & 14 & 0 & -6 & 15 & 3 & -1 & 0 & 0 & 2 \\
5 & 336 & 36 & -8 & -12 & 0 & 6 & 0 & -2 & 0 & 0 & 0 \\
6 & 560 & 50 & 4 & -2 & -8 & 20 & 8 & 4 & 2 & 2 & 0 \\
7 & 1240 & 190 & 36 & 2 & -16 & 40 & 10 & 0 & 1 & 1 & -8 \\
8 & 1512 & 222 & 16 & -18 & 24 & 27 & 3 & -5 & 0 & 0 & -10 \\
9 & 651 & 81 & -5 & -15 & 27 & 6 & 0 & -2 & 0 & 0 & -3 \\
\hline
\end{tabular}

Table 7: Character values of $S_{9}$ on $\widetilde{H}^{i}\left(\mathcal{M}_{\left(3,1^{6}\right)}^{2}\right)$

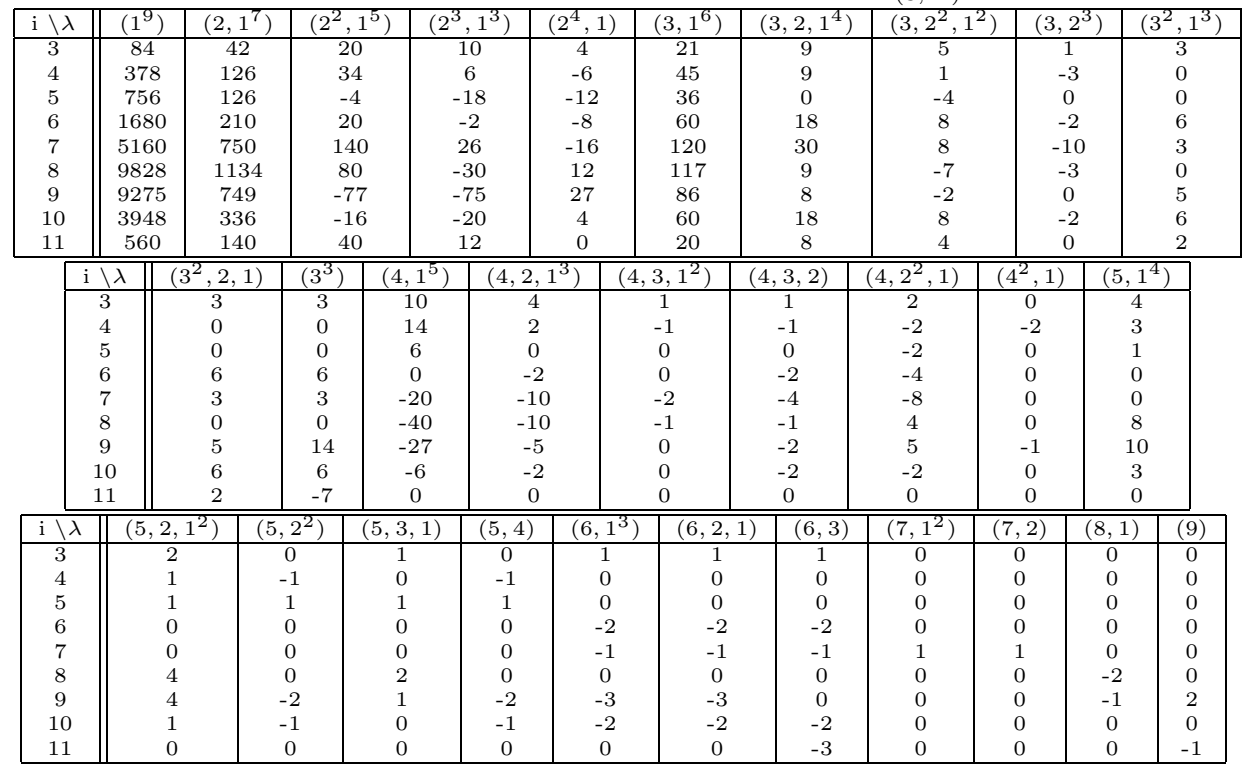


Table 8: Decomposition of the $S_{3}$-module $\widetilde{H}^{i}\left(\mathcal{M}_{(3)}\right)$ into irreducibles

$$
\begin{array}{|c||c|c|c|}
\hline \mathrm{i} \backslash \lambda & \left(1^{3}\right) & (2,1) & (3) \\
\hline 3 & 0 & 0 & 1 \\
\hline
\end{array}
$$

Table 9: Decomposition of the $S_{4}$-module $\widetilde{H}^{i}\left(\mathcal{M}_{(3,1)}\right)$ into irreducibles

\begin{tabular}{|c||c|c|c|c|c|}
\hline $\mathrm{i} \backslash \lambda$ & $\left(1^{4}\right)$ & $\left(2,1^{2}\right)$ & $\left(2^{2}\right)$ & $(3,1)$ & $(4)$ \\
\hline 3 & 0 & 0 & 0 & 1 & 1 \\
4 & 0 & 0 & 0 & 1 & 0 \\
\hline
\end{tabular}

Table 10 : Decomposition of the $S_{5}$-module $\widetilde{H}^{i}\left(\mathcal{M}_{\left(3,1^{2}\right)}\right)$ into irreducibles

\begin{tabular}{|c||c|c|c|c|c|c|c|}
\hline $\mathrm{i} \backslash \lambda$ & $\left(1^{5}\right)$ & $\left(2,1^{3}\right)$ & $\left(2^{2}, 1\right)$ & $\left(3,1^{2}\right)$ & $(3,2)$ & $(4,1)$ & $(5)$ \\
\hline 3 & 0 & 0 & 0 & 0 & 1 & 1 & 1 \\
4 & 0 & 0 & 0 & 1 & 1 & 1 & 0 \\
5 & 0 & 0 & 0 & 1 & 0 & 0 & 0 \\
\hline
\end{tabular}

Table 11 : Decomposition of the $S_{6}$-module $\widetilde{H}^{i}\left(\mathcal{M}_{\left(3,1^{3}\right)}\right)$ into irreducibles

\begin{tabular}{|c||c|c|c|c|c|c|c|c|c|c|c|}
\hline $\mathrm{i} \backslash \lambda$ & $\left(1^{6}\right)$ & $\left(2,1^{4}\right)$ & $\left(2^{2}, 1^{2}\right)$ & $\left(2^{3}\right)$ & $\left(3,1^{3}\right)$ & $(3,2,1)$ & $\left(3^{2}\right)$ & $\left(4,1^{2}\right)$ & $(4,2)$ & $(5,1)$ & $(6)$ \\
\hline 3 & 0 & 0 & 0 & 0 & 0 & 0 & 1 & 0 & 1 & 1 & 1 \\
4 & 0 & 0 & 0 & 0 & 0 & 1 & 1 & 1 & 1 & 1 & 0 \\
5 & 0 & 0 & 0 & 0 & 1 & 1 & 0 & 1 & 0 & 0 & 0 \\
6 & 0 & 0 & 0 & 0 & 1 & 0 & 1 & 0 & 0 & 1 & 0 \\
7 & 0 & 0 & 0 & 0 & 0 & 0 & 1 & 0 & 0 & 1 & 0 \\
\hline
\end{tabular}

Table 12 : Decomposition of the $S_{7}$-module $\widetilde{H}^{i}\left(\mathcal{M}_{\left(3,1^{4}\right)}\right)$ into irreducibles

\begin{tabular}{|c||c|c|c|c|c|c|c|c|}
\hline $\mathrm{i} \backslash \lambda$ & $\left(1^{7}\right)$ & $\left(2,1^{5}\right)$ & $\left(2^{2}, 1^{3}\right)$ & $\left(2^{3}, 1\right)$ & $\left(3,1^{4}\right)$ & $\left(3,2,1^{2}\right)$ & $\left(3,2^{2}\right)$ \\
\hline 3 & 0 & 0 & 0 & 0 & 0 & 0 & 0 \\
4 & 0 & 0 & 0 & 0 & 0 & 0 & 0 \\
5 & 0 & 0 & 0 & 0 & 0 & 1 & 0 \\
6 & 0 & 0 & 0 & 0 & 1 & 1 & 0 \\
7 & 0 & 0 & 0 & 0 & 1 & 0 & 0 \\
8 & 0 & 0 & 0 & 0 & 0 & 0 & 0 \\
\hline $\mathrm{i} \backslash \lambda$ & $\left(3^{2}, 1\right)$ & $\left(4,1^{3}\right)$ & $(4,2,1)$ & $(4,3)$ & $\left(5,1^{2}\right)$ & $(5,2)$ & $(6,1)$ & $(7)$ \\
\hline 3 & 0 & 0 & 0 & 1 & 0 & 1 & 1 & 1 \\
4 & 1 & 0 & 1 & 1 & 1 & 1 & 1 & 0 \\
5 & 1 & 1 & 1 & 0 & 1 & 0 & 0 & 0 \\
6 & 1 & 1 & 0 & 1 & 1 & 1 & 1 & 0 \\
7 & 2 & 0 & 1 & 2 & 2 & 2 & 2 & 0 \\
8 & 1 & 0 & 1 & 1 & 1 & 1 & 1 & 0 \\
\hline
\end{tabular}

Table 13 : Decomposition of the $S_{8}$-module $\widetilde{H}^{i}\left(\mathcal{M}_{\left(3,1^{5}\right)}\right)$ into irreducibles

\begin{tabular}{|c||c|c|c|c|c|c|c|c|c|c|c|}
\hline i $\backslash \lambda$ & $\left(1^{8}\right)$ & $\left(2,1^{6}\right)$ & $\left(2^{2}, 1^{4}\right)$ & $\left(2^{3}, 1^{2}\right)$ & $\left(2^{4}\right)$ & $\left(3,1^{5}\right)$ & $\left(3,2,1^{3}\right)$ & $\left(3,2^{2}, 1\right)$ & $\left(3^{2}, 1^{2}\right)$ & $\left(3^{2}, 2\right)$ & $\left(4,1^{4}\right)$ \\
\hline 3 & 0 & 0 & 0 & 0 & 0 & 0 & 0 & 0 & 0 & 0 & 0 \\
4 & 0 & 0 & 0 & 0 & 0 & 0 & 0 & 0 & 0 & 0 & 0 \\
5 & 0 & 0 & 0 & 0 & 0 & 0 & 0 & 0 & 1 & 0 & 0 \\
6 & 0 & 0 & 0 & 0 & 0 & 0 & 1 & 0 & 1 & 1 & 1 \\
7 & 0 & 0 & 0 & 0 & 0 & 1 & 1 & 0 & 1 & 2 & 1 \\
8 & 0 & 0 & 0 & 0 & 0 & 1 & 0 & 0 & 3 & 1 & 0 \\
9 & 0 & 0 & 0 & 0 & 0 & 0 & 0 & 0 & 2 & 0 & 0 \\
\hline
\end{tabular}

Table 14 : Decomposition of the $S_{9}$-module $\widetilde{H}^{i}\left(\mathcal{M}_{\left(3,1^{6}\right)}^{2}\right)$ into irreducibles

\begin{tabular}{|c||c|c|c|c|c|c|c|c|c|c|}
\hline $\mathrm{i} \backslash \lambda$ & $\left(1^{9}\right)$ & $\left(2,1^{7}\right)$ & $\left(2^{2}, 1^{5}\right)$ & $\left(2^{3}, 1^{3}\right)$ & $\left(2^{4}, 1\right)$ & $\left(3,1^{5}\right)$ & $\left(3,2,1^{4}\right)$ & $\left(3,2^{2}, 1^{2}\right)$ & $\left(3,2^{3}\right)$ & $\left(3^{2}, 1^{3}\right)$ \\
\hline 3 & 0 & 0 & 0 & 0 & 0 & 0 & 0 & 0 & 0 & 0 \\
4 & 0 & 0 & 0 & 0 & 0 & 0 & 0 & 0 & 0 & 0 \\
5 & 0 & 0 & 0 & 0 & 0 & 0 & 0 & 0 & 0 & 0 \\
6 & 0 & 0 & 0 & 0 & 0 & 0 & 0 & 0 & 0 & 1 \\
7 & 0 & 0 & 0 & 0 & 0 & 0 & 1 & 0 & 0 & 1 \\
8 & 0 & 0 & 0 & 0 & 0 & 1 & 1 & 0 & 0 & 2 \\
9 & 0 & 0 & 0 & 0 & 0 & 1 & 0 & 0 & 0 & 4 \\
10 & 0 & 0 & 0 & 0 & 0 & 0 & 0 & 0 & 0 & 0 \\
11 & 0 & 0 & 0 & 0 & 0 & 0 & 0 & 0 & 0 & 0 \\
\hline
\end{tabular}




\begin{tabular}{|c||c|c|c|c|c|c|c|c|c|c|c|}
\hline $\mathrm{i} \backslash \lambda$ & $\left(5,2,1^{2}\right)$ & $\left(5,2^{2}\right)$ & $(5,3,1)$ & $(5,4)$ & $\left(6,1^{3}\right)$ & $(6,2,1)$ & $(6,3)$ & $\left(7,1^{2}\right)$ & $(7,2)$ & $(8,1)$ & $(9)$ \\
\hline 3 & 0 & 0 & 0 & 0 & 0 & 0 & 1 & 0 & 1 & 1 & 1 \\
4 & 0 & 0 & 1 & 0 & 0 & 1 & 1 & 1 & 1 & 1 & 0 \\
5 & 1 & 0 & 1 & 0 & 1 & 1 & 0 & 1 & 0 & 0 & 0 \\
6 & 1 & 0 & 2 & 1 & 1 & 1 & 2 & 1 & 1 & 1 & 0 \\
7 & 2 & 2 & 7 & 3 & 1 & 5 & 5 & 3 & 3 & 2 & 0 \\
8 & 7 & 5 & 9 & 3 & 4 & 8 & 4 & 3 & 3 & 1 & 0 \\
9 & 8 & 4 & 6 & 1 & 5 & 5 & 2 & 2 & 1 & 0 & 0 \\
10 & 3 & 1 & 3 & 1 & 2 & 2 & 2 & 1 & 1 & 1 & 0 \\
11 & 0 & 0 & 1 & 1 & 0 & 1 & 1 & 0 & 1 & 1 & 0 \\
\hline
\end{tabular}

It is easy to see that, for even $d$, the irreducible indexed by $\lambda$ occurs in the cohomology of $\mathcal{M}_{\left(k, 1^{n-k}\right)}^{d}$ only if all parts of $\lambda$ are greater than or equal to $k$.

In the next tables we list a few sample values for the cohomology representations of $S_{2 \ell}$ on the manifolds $\mathcal{M}_{\left(2^{\ell}\right)}^{2}$. We note that for arbitrary $k$, the lowest nonvanishing cohomology of $\mathcal{M}_{\left(k^{\ell}\right)}^{2}$ is always a transitive permutation module. Also for all $k$ the top non-vanishing cohomology of $\mathcal{M}_{\left(k^{\ell}\right)}^{2}$ is (as an $S_{k \ell}$-module) isomorphic to the unique non-vanishing homology module of the lattice $\Pi_{\left(k^{\ell}\right)}$. In particular, for $k=2$ its dimension is always given by an Euler number.

Before we start with the tables we state one result on the behaviour of the character values of $S_{k \ell}$ on the cohomology of $\mathcal{M}_{\left(k^{\ell}\right)}^{2}$.

Proposition 5.1. Let $\beta_{t, k \ell}$ denote the Betti number of the manifold $\mathcal{M}_{\left(k^{\ell}\right)}^{2}$ (resp. $\mathcal{M}_{\left(k^{\ell}\right)}^{d}$ for arbitrary even $\left.d\right)$ in degree $\ell(2 k-1)-t-1$, (resp. in degree $\ell(d k-1)-$ $t(d-1)-1)$, for $t=1, \ldots, \ell$.

Then these numbers reappear as character values of the cohomology of $\mathcal{M}_{\left(k^{\ell}\right)}^{2}$ (resp. $\left.\mathcal{M}_{\left(k^{\ell}\right)}^{d}\right)$ as follows:

(1) The character value on a kn-cycle $\left(k n, 1^{k \ell}\right)$ in cohomology degree $(\ell+n)(2 k-1)-(t+n)-1($ resp. $(l+n)(d k-1)-(t+n)(d-1)-1)$ is $\beta_{t, k l}$, for $1 \leq t \leq \ell$ (i.e., in the first $\ell$ degrees of cohomology, starting with the lowest degree). This character value is zero in all other cohomology degrees.

(2) The character value on a $(k n+1)$-cycle $\left(k n+1,1^{k \ell-1}\right)$ in cohomology degree $(\ell+n)(2 k-1)-t-1($ resp. $(\ell+n)(d k-1)-t(d-1)-1)$ is $(-1)^{n} \beta_{t, k \ell}$ for $1 \leq t \leq \ell$, and zero in all other degrees.

Proof. Recall that the reduced cohomology of $\mathcal{M}_{\left(k^{\ell}\right)}^{2}$ (resp. $\left.\mathcal{M}_{\left(k^{\ell}\right)}^{d}\right)$ in degree $\ell(2 k-1)-t-1$ (resp. $\ell(d k-1)-t(d-1)-1)$ is given by the Whitney homology of the $k$-divisible lattice $\Pi_{\left(k^{\ell}\right)}$ in degree $\ell+1-t$, for $1 \leq t \leq \ell$.

Now use [Theorem 4.8 (iii)] and the defining equation in [C-H-R] (see also [Su, Theorem 5.1]) for the top homology of the lattice $\Pi_{\left(k^{\ell}\right)}$. The Frobenius characteristic of the Whitney homology $W H_{\ell+1-t}\left(\Pi_{\left(k^{\ell}\right)}\right)$ may thus be rewritten as the degree $(k \ell)$-term in the plethysm

$$
(-1)^{\ell} e_{t}\left[\sum_{i \geq 1}(-1)^{i} \pi_{i}\left[\sum_{j \geq 1} h_{k j}\right]\right],
$$

where

$$
\pi_{i}=\frac{1}{i} \sum_{d \mid i} \mu(d)(-1)^{i-i / d} p_{d}^{i / d}
$$

is the Frobenius characteristic of the top homology of the partition lattice $\Pi_{i}$.

This expression will greatly simplify our calculations. As in [Macd], $p_{n}$ denotes the $n$th power sum symmetric function. Note first that by definition of the Betti 
number $\beta_{t, k \ell}$, the coefficient of $p_{1}^{k \ell}$ in the above expression is

$$
\frac{\beta_{t, k \ell}}{(k \ell) !}
$$

Write $Y$ for the power series $\sum_{j \geq 1} \frac{p_{1}^{k j}}{(k j) !}$. Note that $-\log (1+Y)=\sum_{i \geq 1}(-1)^{i} \frac{Y^{i}}{i}$. From standard properties of the plethysm operation we deduce the following power series expansion, for $1 \leq t \leq \ell$ :

$$
(-\log (1+Y))^{t}=\sum_{\ell \geq 1}(-1)^{\ell} \beta_{t, k \ell} \frac{t !}{(k \ell) !} p_{1}^{k \ell}
$$

We are now ready to compute the character values on cycles congruent to 0 and 1 modulo $k$.

For the first result, we need to compute the coefficient of the symmetric function $p_{k n} p_{1}^{k \ell}$ in the Whitney homology $W H_{\ell+1-t}\left(\Pi_{\left(k^{\ell+n}\right)}\right)$, whose characteristic is

$$
(-1)^{\ell+n} e_{t+n}\left[\sum_{i \geq 1}(-1)^{i} \pi_{i}\left[\sum_{j \geq 1} h_{k j}\right]\right]
$$

The result will be established if we show that this coefficient is $\frac{1}{k n} \frac{1}{(k \ell) !} \beta_{t, k \ell}$ if $1 \leq$ $t \leq \ell$, and is zero otherwise.

From (A), the only terms of $\pi_{i}$ that we need to consider are those involving $p_{1}^{i}, i \geq 1$, and $p_{i}, i \geq 2$. In the innermost plethysm, the only terms relevant to the computation are those corresponding to the cycle-types $\left(1^{k j}\right)$ and $\left(k j, 1^{k m}\right)$. These terms are accounted for by the expression $Y+\sum_{j \geq 1} \frac{p_{k j}}{k j}(1+Y)$.

Hence the contributions to a $(k n)$-cycle come only from the plethysm of $(-1)^{\ell+n} e_{t+n}$ with

$$
\sum_{i \geq 1} \frac{(-1)^{i}}{i}\left(Y+\sum_{j \geq 1} \frac{p_{k j}}{k j}(1+Y)\right)^{i}-\sum_{i \geq 2} \frac{\mu(i)}{i}\left(Y\left[p_{i}\right]+\sum_{j \geq 1} \frac{p_{k j i}}{k j i}\right)
$$

Observe that we can discard powers of $\sum_{j \geq 1} \frac{p_{k j}}{k j}$ greater than 1 , and hence the terms $Y\left[p_{i}\right]$ for $i \geq 2$. Further inspection shows that terms of the form $p_{k j}$ vanish for $j \geq 2$, and hence the contributions to a $(k n)$-cycle come only from the plethysm of $(-1)^{\ell+n} e_{t+n}$ with

$$
-\log (1+Y)-\frac{p_{k}}{k}
$$

It is now clear that to obtain a $(k n)$-cycle, $t$ must be nonnegative. In this case the only terms one need consider in the power-sum expansion of the elementary symmetric function $e_{t+n}$ are those corresponding to the cycle-types $\left(1^{t+n}\right)$ and $\left(n, 1^{t}\right)$, the latter occurring only if $n \geq 2$.

Evaluating the plethysm and again discarding the terms in $\log (1+Y)\left[p_{n}\right]$, we find that we need the coefficient of $p_{k n} p_{1}^{k \ell}$ in the expression $(-1)^{\ell+n}\left(Z_{1}+Z_{2}\right)$, where

$$
Z_{1}=-\frac{1}{(t+n-1) !} \frac{p_{k}}{k}(-\log (1+Y))^{t+n-1}
$$

and $Z_{2}$ is nonzero only for $n \geq 2$, in which case we have

$$
Z_{2}=\frac{1}{t !}(-1)^{n}(-\log (1+Y))^{t} \frac{p_{n k}}{n k} \text {. }
$$


Now the result follows easily from the identity (B).

For the second result, we need the coefficient of $p_{k n+1} p_{1}^{k \ell-1}$ in the characteristic of the Whitney homology $W H_{\ell+n+1-t}\left(\Pi_{\left(k^{\ell+n}\right)}\right)$, i.e., in

$$
(-1)^{\ell+n} e_{t}\left[\sum_{i \geq 1}(-1)^{i} \pi_{i}\left[\sum_{j \geq 1} h_{k j}\right]\right] .
$$

The result will be established if we show that this coefficient equals

$$
(-1)^{n} \frac{1}{k n+1} \frac{1}{(k \ell-1) !} \beta_{t, k \ell}
$$

if $1 \leq t \leq \ell$, and is zero otherwise.

Since all terms in the innermost plethysm have degree at least $k$, the only way to obtain the required cycle-type from the plethysm is via terms of the form $p_{k n+1}$ from the innermost plethysm, and only terms involving powers of $p_{1}$ from the other factors.

Hence we need only consider the plethysm of $(-1)^{\ell+n} e_{t}$ with

$$
-\log (1+Y)+\frac{p_{k n+1}}{k n+1} \frac{\partial Y}{\partial p_{1}}
$$

Discarding the terms involving higher powers of $p_{k n+1}$, we have the plethysm of $(-1)^{\ell+n} e_{t}$ with

$$
-\log (1+Y)+\frac{p_{k n+1}}{k n+1} \sum_{i \geq 1}(-1)^{i} Y^{i-1} \frac{\partial Y}{\partial p_{1}} .
$$

Now we observe that we can discard all terms in the power-sum expansion of $e_{t}$ except for the term $p_{1}^{t}$. Hence we require the coefficient of $p_{k n+1} p_{1}^{k \ell-1}$ in the expression

$$
(-1)^{\ell+n} \frac{1}{(t-1) !}(-\log (1+Y))^{t-1} \frac{p_{k n+1}}{k n+1} \sum_{i \geq 1}(-1)^{i} Y^{i-1} \frac{\partial Y}{\partial p_{1}}
$$

The result now follows by taking the partial derivative of $(B)$ with respect to $p_{1}$.

Table 15 : Character values of $S_{4}$ on $\widetilde{H}^{i}\left(\mathcal{M}_{\left(2^{2}\right)}^{2}\right)$

\begin{tabular}{|c||c|c|c|c|c|}
\hline $\mathrm{i} \backslash \lambda$ & $\left(1^{4}\right)$ & $\left(2,1^{2}\right)$ & $\left(2^{2}\right)$ & $(3,1)$ & $(4)$ \\
\hline 3 & 3 & 1 & 3 & 0 & 1 \\
4 & 2 & 0 & 2 & -1 & 0 \\
\hline
\end{tabular}

Table 16 : Character values of $S_{6}$ on $\widetilde{H}^{i}\left(\mathcal{M}_{\left(2^{3}\right)}^{2}\right)$

\begin{tabular}{|c||c|c|c|c|c|c|c|c|c|c|c|}
\hline $\mathrm{i} \backslash \lambda$ & $\left(1^{6}\right)$ & $\left(2,1^{4}\right)$ & $\left(2^{2}, 1^{2}\right)$ & $\left(2^{3}\right)$ & $\left(3,1^{3}\right)$ & $(3,2,1)$ & $\left(3^{2}\right)$ & $\left(4,1^{2}\right)$ & $(4,2)$ & $(5,1)$ & $(6)$ \\
\hline 5 & 15 & 3 & 3 & 7 & 0 & 0 & 3 & 1 & 1 & 0 & 1 \\
6 & 30 & 2 & 2 & 6 & -3 & -1 & 0 & 0 & 0 & 0 & 0 \\
7 & 16 & 0 & 0 & 0 & -2 & 0 & -2 & 0 & 0 & 1 & 0 \\
\hline
\end{tabular}

Table 17: Character values of $S_{8}$ on $\widetilde{H}^{i}\left(\mathcal{M}_{\left(2^{4}\right)}^{2}\right)$

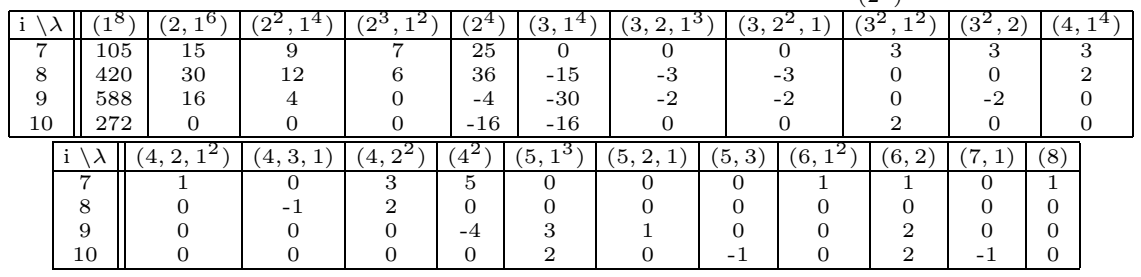


Table 18 : Character values of $S_{10}$ on $\widetilde{H}^{i}\left(\mathcal{M}_{\left(2^{5}\right)}^{2}\right)$

\begin{tabular}{|c|c|c|c|c|c|c|c|c|c|c|c|c|c|c|}
\hline$i \backslash \lambda$ & $\left(1^{10}\right)$ & \multicolumn{2}{|c|}{$\left(2,1^{8}\right)$} & $\left(2^{2}, 1^{6}\right)$ & \multicolumn{2}{|c|}{$\left(2^{3}, 1^{4}\right)$} & $\left(2^{4}, 1^{2}\right)$ & $\left(2^{5}\right)$ & $\left(3,1^{7}\right)$ & $\left(3,2,1^{5}\right)$ & \multicolumn{2}{|c|}{$\left(3,2^{2}, 1^{3}\right)$} & $\left(3,2^{3}, 1\right)$ & $\left(3^{2} \cdot 1^{4}\right)$ \\
\hline 9 & 945 & \multicolumn{2}{|c|}{105} & 45 & \multicolumn{2}{|c|}{21} & 25 & \multirow{2}{*}{81} & \multirow{2}{*}{0} & 0 & \multicolumn{2}{|c|}{0} & 0 & 9 \\
\hline 10 & 6300 & \multicolumn{2}{|c|}{420} & 120 & \multirow{2}{*}{\multicolumn{2}{|c|}{$\begin{array}{l}32 \\
12\end{array}$}} & 36 & & & -15 & \multicolumn{2}{|c|}{-9} & -7 & 6 \\
\hline 11 & 16380 & \multicolumn{2}{|c|}{588} & \multirow{2}{*}{108} & & & -4 & 140 & -420 & -30 & -1 & & -6 & 6 \\
\hline 12 & 18960 & 27 & & & & 0 & -16 & $\begin{array}{l}-20 \\
-80\end{array}$ & -588 & -16 & -4 & & 0 & 24 \\
\hline 13 & 7936 & 0 & & 0 & & 0 & 0 & 0 & -272 & 0 & 0 & & 0 & 16 \\
\hline$\overline{i \backslash \lambda}$ & $\overline{\left(\overline{\left(3^{2}, 2\right.}\right.}$ & $\left(1^{2}\right)$ & $\left(3^{2}\right.$, &, $\left.2^{2}\right)$ & $33^{3}, 1$ & $\overline{(4,}$ & $\overline{(4,2}$ & $\left.2,1^{4}\right)$ & $\left(4,2^{2}, 1^{2}\right)$ & $\left(4,2^{3}\right)$ & $\overline{(4,3,}$ & $\left.1^{3}\right)$ & $(4,3,2,1)$ & $\left(4,3^{2}\right)$ \\
\hline 9 & 3 & & & 9 & 0 & 1 & 5 & 3 & 3 & 7 & 0 & & 0 & 3 \\
\hline 10 & 0 & & & 6 & -9 & 3 & 0 & 2 & 2 & 6 & -3 & & -1 & 0 \\
\hline 11 & 0 & & & -6 & 0 & 1 & 6 & 0 & 0 & 0 & -2 & & 0 & -2 \\
\hline 12 & 2 & & & -4 & 6 & ( & 0 & 0 & 0 & 0 & 0 & & 0 & 0 \\
\hline 13 & 0 & & & 0 & -2 & ( & 0 & 0 & 0 & 0 & 0 & & 0 & 0 \\
\hline$i \backslash \lambda$ & $\left(4^{2}, 1^{2}\right.$ & $\overline{(4)}$ & 2, 2) & $\left(5,1^{5}\right)$ & \begin{tabular}{l|l}
) & $(5$
\end{tabular} & $2,1^{3}$ & $\left(5,2^{2}\right.$, & & $\left.3,3,1^{2}\right)$ & $(5,3,2)$ & $(5,4,1)$ & $\left(5^{2}\right)$ & $\left(6,1^{4}\right)$ & $\left(6,2,1^{2}\right)$ \\
\hline 9 & 5 & & 5 & 0 & & 0 & 0 & & 0 & 0 & 0 & 5 & 3 & 1 \\
\hline 10 & 0 & & 0 & 0 & & 0 & 0 & & 0 & 0 & 0 & 0 & 2 & 0 \\
\hline 11 & -4 & & -4 & 15 & & 3 & 3 & & 0 & 0 & 1 & 0 & 0 & 2 \\
\hline 12 & 0 & & 0 & 30 & & 2 & 2 & & -3 & -1 & 0 & 0 & 0 & 2 \\
\hline 13 & 0 & & 0 & 16 & & 0 & 0 & & -2 & 0 & 0 & -4 & 0 & 0 \\
\hline & i & $\lambda$ & $6,2^{2}$ & $(6,3$ & $3,1)$ & $(6,4)$ & $\left(7,1^{3}\right)$ & $\overline{(7,2,}$ & $(7,3)$ & $\left(8,1^{2}\right)$ & $(8,2)$ & $(9,10$ & (10) & \\
\hline & 9 & & 3 & 0 & & 1 & 0 & 0 & 0 & 1 & 1 & 0 & 1 & \\
\hline & 1 & & 2 & -1 & & 0 & 0 & 0 & 0 & 0 & 0 & 0 & 0 & \\
\hline & 1 & & 4 & 0 & & 0 & 0 & 0 & 0 & 0 & 0 & 0 & 0 & \\
\hline & 1 & & 4 & 0 & & 0 & -3 & -1 & 0 & 0 & 0 & 0 & 0 & \\
\hline & 1 & & 0 & 0 & & 0 & -2 & 0 & 1 & 0 & 0 & 1 & 0 & \\
\hline
\end{tabular}

Table 19 : Decomposition of the $S_{4}$-module $\widetilde{H}^{i}\left(\mathcal{M}_{\left(2^{2}\right)}\right)$ into irreducibles

$$
\begin{array}{|c||c|c|c|c|c|}
\hline \mathrm{i} \backslash \lambda & \left(1^{4}\right) & \left(2,1^{2}\right) & \left(2^{2}\right) & (3,1) & (4) \\
\hline 3 & 0 & 0 & 1 & 0 & 1 \\
4 & 0 & 0 & 1 & 0 & 0 \\
\hline
\end{array}
$$

Table 20 : Decomposition of the $S_{6}$-module $\widetilde{H}^{i}\left(\mathcal{M}_{\left(2^{3}\right)}\right)$ into irreducibles

\begin{tabular}{|c||c|c|c|c|c|c|c|c|c|c|c|}
\hline $\mathrm{i} \backslash \lambda$ & $\left(1^{6}\right)$ & $\left(2,1^{4}\right)$ & $\left(2^{2}, 1^{2}\right)$ & $\left(2^{3}\right)$ & $\left(3,1^{3}\right)$ & $(3,2,1)$ & $\left(3^{2}\right)$ & $\left(4,1^{2}\right)$ & $(4,2)$ & $(5,1)$ & $(6)$ \\
\hline 5 & 0 & 0 & 0 & 1 & 0 & 0 & 0 & 0 & 1 & 0 & 1 \\
6 & 0 & 0 & 0 & 1 & 0 & 1 & 0 & 0 & 1 & 0 & 0 \\
7 & 0 & 0 & 0 & 0 & 0 & 1 & 0 & 0 & 0 & 0 & 0 \\
\hline
\end{tabular}

Table 21 : Decomposition of the $S_{8}$-module $\widetilde{H}^{i}\left(\mathcal{M}_{\left(2^{4}\right)}\right)$ into irreducibles

\begin{tabular}{|c||c|c|c|c|c|c|c|c|c|c|c|c|}
\hline $\mathrm{i} \backslash \lambda$ & $\left(1^{8}\right)$ & $\left(2,1^{6}\right)$ & $\left(2^{2}, 1^{4}\right)$ & $\left(2^{3}, 1^{2}\right)$ & $\left(2^{4}\right)$ & $\left(3,1^{5}\right)$ & $\left(3,2,1^{3}\right)$ & $\left(3,2^{2}, 1\right)$ & $\left(3^{2}, 1^{2}\right)$ & $\left(3^{2}, 2\right)$ & $\left(4,1^{4}\right)$ \\
\hline 7 & 0 & 0 & 0 & 0 & 1 & 0 & 0 & 0 & 0 & 0 & 0 \\
8 & 0 & 0 & 0 & 0 & 1 & 0 & 0 & 1 & 1 & 0 & 0 \\
9 & 0 & 0 & 0 & 0 & 0 & 0 & 0 & 2 & 1 & 1 & 0 \\
10 & 0 & 0 & 0 & 0 & 0 & 0 & 0 & 1 & 0 & 1 & 0 \\
\hline \multicolumn{2}{c||}{$\mathrm{i} \backslash \lambda$} & $\left(4,2,1^{2}\right)$ & $(4,3,1)$ & $\left(4,2^{2}\right)$ & $\left(4^{2}\right)$ & $\left(5,1^{3}\right)$ & $(5,2,1)$ & $(5,3)$ & $\left(6,1^{2}\right)$ & $(6,2)$ & $(7,1)$ & $(8)$ \\
\hline 7 & 0 & 0 & 1 & 1 & 0 & 0 & 0 & 0 & 1 & 0 & 1 \\
8 & 0 & 1 & 2 & 1 & 0 & 1 & 0 & 0 & 1 & 0 & 0 \\
9 & 1 & 2 & 1 & 0 & 0 & 1 & 0 & 0 & 0 & 0 & 0 \\
10 & 1 & 1 & 0 & 0 & 0 & 0 & 0 & 0 & 0 & 0 & 0 \\
\hline
\end{tabular}

Table 22 : Decomposition of the $S_{10}$-module $H^{i}\left(\mathcal{M}_{\left(2^{5}\right)}\right)$ into irreducibles

\begin{tabular}{|c||c|c|c|c|c|c|c|c|c|c|c|}
\hline $\mathrm{i} \backslash \lambda$ & $\left(1^{10}\right)$ & $\left(2,1^{8}\right)$ & $\left(2^{2}, 1^{6}\right)$ & $\left(2^{3}, 1^{4}\right)$ & $\left(2^{4}, 1^{2}\right)$ & $\left(2^{5}\right)$ & $\left(3,1^{7}\right)$ & $\left(3,2,1^{5}\right)$ & $\left(3,2^{2}, 1^{3}\right)$ & $\left(3,2^{3}, 1\right)$ & $\left(3^{2}, 1^{4}\right)$ \\
\hline 9 & 0 & 0 & 0 & 0 & 0 & 1 & 0 & 0 & 0 & 0 & 0 \\
10 & 0 & 0 & 0 & 0 & 0 & 1 & 0 & 0 & 0 & 1 & 0 \\
11 & 0 & 0 & 0 & 0 & 0 & 0 & 0 & 0 & 0 & 2 & 0 \\
12 & 0 & 0 & 0 & 0 & 0 & 0 & 0 & 0 & 0 & 2 & 0 \\
13 & 0 & 0 & 0 & 0 & 0 & 0 & 0 & 0 & 0 & 1 & 0 \\
\hline
\end{tabular}

\begin{tabular}{|c|c|c|c|c|c|c|c|c|c|c|c|c|c|c|}
\hline$\overline{i \backslash \backslash \lambda}$ & $\left(3^{2}, 2,1^{2}\right)$ & \multicolumn{2}{|c|}{$\left(3^{2}, 2^{2}\right)$} & $\left(3^{3}, 1\right)$ & $\left(4,1^{6}\right)$ & \multicolumn{2}{|c|}{$\left(4,2,1^{4}\right)$} & \multicolumn{2}{|c|}{$\left(4,2^{2}, 1^{2}\right)$} & \begin{tabular}{l|l}
)$\left(4,2^{3}\right)$ \\
\end{tabular} & \multicolumn{2}{|c|}{$\left(4,3,1^{3}\right)$} & $(4,3,2,1)$ & $\left(4,3^{2}\right)$ \\
\hline 9 & 0 & 0 & & 0 & 0 & & & & 0 & 1 & 0 & & & \\
\hline 10 & 1 & 0 & & 0 & 0 & 0 & & & 0 & 2 & 0 & & 2 & 0 \\
\hline 11 & 2 & 2 & & 1 & 0 & 0 & & & 2 & 2 & 1 & & 5 & 2 \\
\hline 12 & 2 & 3 & & 2 & 0 & 0 & & & 3 & 2 & 2 & & 6 & 3 \\
\hline 13 & 1 & 1 & & 1 & 0 & 0 & & & 1 & 1 & 1 & & 3 & 1 \\
\hline $\mathrm{i} \backslash \lambda$ & $\left(4^{2}, 1^{2}\right)$ & 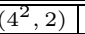 & (5,1 & \begin{tabular}{l|l}
$5)$ & $(5$ \\
\end{tabular} & $\left., 2,1^{3}\right)$ & $\overline{\left(5,2^{2},\right.}$ & & $\overline{5,3,1}$ & & $(5,3,2)$ & $\overline{(5,4,1)}$ & $\left(5^{2}\right)$ & $\left(6,1^{4}\right)$ & $\left(6,2,1^{2}\right)$ \\
\hline 9 & 0 & 1 & 0 & & 0 & 0 & & 0 & & 0 & 0 & 0 & 0 & 0 \\
\hline 10 & 0 & 2 & 0 & & 0 & 1 & & 1 & & 1 & 1 & 0 & 0 & 0 \\
\hline 11 & 2 & 2 & 0 & & 0 & 3 & & 3 & & 3 & 2 & 0 & 0 & 1 \\
\hline 12 & 3 & 2 & 0 & & 1 & 3 & & 3 & & 3 & 2 & 0 & 0 & 1 \\
\hline 13 & 1 & 1 & 0 & & 1 & 1 & & 1 & & 1 & 1 & 0 & 0 & 0 \\
\hline & $\mathrm{i} \backslash \lambda$ & $\overline{\left(6,2^{2}\right)}$ & & (3,1) & $\overline{(6,4)}$ & $\left(7,1^{3}\right)$ & $(7,2$, & & $(7,3)$ & $\left(8,1^{2}\right)$ & $\begin{array}{l}(8,2) \\
\end{array}$ & $\begin{array}{l}(9,1) \\
\end{array}$ & \begin{tabular}{|l|l|}
) & $(10)$ \\
\end{tabular} & \\
\hline & \begin{tabular}{|l|}
9 \\
\end{tabular} & 1 & & 0 & 1 & 0 & 0 & & 0 & 0 & 1 & 0 & $\begin{array}{ll}1 \\
\end{array}$ & \\
\hline & 10 & 2 & & 1 & 1 & 0 & 1 & & 0 & 0 & 1 & 0 & 0 & \\
\hline & 11 & 1 & & 2 & 0 & 0 & 1 & & 0 & 0 & 0 & 0 & 0 & \\
\hline & 12 & 0 & & 1 & 0 & 0 & 0 & & 0 & 0 & 0 & 0 & 0 & \\
\hline & 13 & 0 & & 0 & 0 & 0 & 0 & & 0 & 0 & 0 & 0 & 0 & \\
\hline
\end{tabular}




\section{REFERENCES}

[Ar1] Arnol'd, V.I.: The cohomology ring of the colored braid group. Math. Notes 5, 138140 (1969) MR 39:3529

[Ar2] Arnol'd, V.I.: Topological invariants of algebraic functions. Trans. Moscow Math. Soc. 21, 30-52 (1970) MR 43:1991

[Ar3] Arnol'd, V.I.: The spaces of functions with mild singularities. Funktsional. Anal. i Prilozhen. 3, 1-10 (1989). MR 90m:58016 English translation in Functional Anal. Appl. 23 (1989)

[Ba] Baclawski, K.: Whitney numbers of geometric lattices. Adv. in Math. 16, 125-138 (1975) MR 52:7933

[Bj1] Björner, A.: Shellable and Cohen-Macaulay partially ordered sets. Trans. Amer. Math. Soc. 260, 159-183 (1980) MR 81i:06001

[Bj2] Björner, A.: On the homology of geometric lattices, Algebra Universalis 14, 107-128 (1982). MR 83d:05029

[Bj3] Björner, A.: Some combinatorial and algebraic properties of Coxeter complexes and Tits buildings, Adv. in Math. 52, 173-212 (1984). MR 85m:52003

[Bj4] Björner, A.: Subspace arrangements. In: Proceedings of the 1st European Congress of Mathematics (Paris 1992), ed. A. Joseph, R. Rentschler, Basel, Boston: Birkhäuser, 321-370 (1994). MR 96h:52012

[Bj-Lo-Y] Björner, A., Lovász, L., Yao, A.: Linear decision trees: volume estimates and topological bounds. In: Proc. 24th ACM Symp. on Theory of Computing, pp. 170-177. New York: ACM Press 1992

[Bj-Lo] Björner, A., Lovász, L.: Linear decision trees, subspace arrangements and Möbius functions. J. Amer. Math. Soc. 7, 677-706 (1994) MR 95e:52024

[Bj-Wa] Björner, A., Wachs, M.: Shellable nonpure complexes and posets, I, Trans. Amer. Math. Soc. 348 1299-1327 (1996). MR 96i:06008

[Bj-Wal] Björner, A., Walker, J.W.: A homotopy complementation formula for partially ordered sets. European J. Combin. 4, 11-19 (1983) MR 84f:06003

[Bj-We] Björner, A., Welker, V.: Homology of the " $k$-equal" manifolds and related partition lattices. Adv. in Math. 110(2), 277-313 (1995). MR 95m:52029

[Bo-Ka] Bousfield, M., Kan, D. M.: Homotopy Limits, Completions and Localizations. (Lecture Notes in Mathematics, Vol. 304). Berlin, Heidelberg, New York: Springer 1972 MR 51:1825

[Bre] Bredon, G.: Compact Transformation Groups. New York, London: Academic Press 1972 MR 54:1265

[Bri] Brieskorn, E.: Sur les groupes de tresses. In: Séminaire Bourbaki 1971/72, pp. 21-44. Berlin, Heidelberg, New York: Springer 1973 MR 54:10660

[C-H-R] Calderbank, A., Hanlon, P., Robinson, R.: Partitions into even and odd block sizes and some unusual characters of the symmetric groups. Proc. London Math. Soc. 53(3), 288-320 (1986) MR 87m:20042

[Co-La-Ma] Cohen, F.L., Lada, T.J., May, J.P., The Homology of Iterated Loop Spaces. (Lecture Notes in Mathematics, Vol. 533). Berlin, Heidelberg, New York: Springer 1976 MR $\mathbf{5 5 : 9 0 9 6}$

[Co-Lu] Cohen, F.L., Lusk, E.L.: Configuration-like spaces and the Borsuk-Ulam Theorem, Proc. Amer. Math. Soc. 56, 313-317 (1976) MR 54:13899

[Ep] Epshtein,S.I.: Fundamental groups of coprime polynomials. Functional Anal. Appl. 7,82-83 (1973) MR 49:9865

[Fa-Ne] Fadell, E., Neuwirth, L.: Configuration spaces. Math. Scand. 10, 111-118 (1962) MR 25:4537

[Fo-Ne] Fox, R., Neuwirth, L.: The braid groups. Math. Scand. 10, 119-126 (1962) MR 27:742

[Fu] Fuks, D.: Cohomologies of the braid groups mod 2. Functional Anal. Appl. 4, 143-151 (1970) MR 43:226

[Gab-Zis] Gabriel, P., Zisman, M.: Calculus of Fractions and Homotopy Theory. (Ergebnisse der Mathematik und ihrer Grenzgebiete, Vol. 35). Berlin, Heidelberg, New York: Springer 1967 MR 35:1019 
[Go-MacP] Goresky, M., MacPherson, R.: Stratified Morse Theory. (Ergebnisse der Mathematik und ihrer Grenzgebiete, Vol. 14). Berlin, Heidelberg, New York: Springer 1988 MR 90d:57039

[Gu-Ko-Y] Guest, M., Kozlowski, A., Yamaguchi, K.: The topology of spaces of coprime polynomials. Math. Z. 217, No. 3, 435-446 (1994) MR 95:05

[Ha1] Hanlon, P.: The fixed-point partition lattices, Pacific J. Math. 96, 319-341 (1981) MR 83d:06010

[Ha2] Hanlon, P.: The generalized Dowling lattices. Trans. AMS 325(1), 1-37 (1991) MR 91h:06011

[Hu] Hu, Y.: Homology of subspace arrangements. Proc. Amer. Math. Soc. 122, 285-290 (1994). MR 94k:52020

[Je] Jewell, K.: Complements of sphere and subspace arrangements. Topology and its Appl. 56, No. 3, 199-214 (1994) MR 95a:52017

[Le-So] Lehrer, G., Solomon, L.: On the action of the symmetric group on the cohomology of the complement of its reflecting hyperplanes. J. Algebra 104, 410-424 (1986) MR 88a:32017

[Macd] Macdonald, I.: Symmetric functions and Hall polynomials. Oxford University Press 1979 MR 84g:05003

[Me] Merkov, A.B.: Finite-order invariants of ornaments (Preprint 1994).

$[\mathrm{Mu}] \quad$ J. R. Munkres, Elements of Algebraic Topology, Addison-Wesley (1984) MR 85m:55001

[O-So] Orlik, P., Solomon, L.: Combinatorics and topology of complements of hyperplanes. Invent. Math. 56, 167-189 (1980) MR 81e:32015

[O-T] Orlik, P., Terao, H.: Arrangements of Hyperplanes. (Grundlehren der mathematischen Wissenschaften, Vol. 300). Berlin, Heidelberg, New York: Springer 1992 MR 94e:52014

[Sa] Sagan, B.: Shellability of exponential structures. Order 3, 47-54 (1986) MR 87j:05020

[Se] Segal, G.: Classifying spaces and spectral sequences. Publ. Math. I.H.E.S. 34, 105112 (1968) MR 38:718

[So] Solomon, L.: A decomposition of the group algebra of a finite Coxeter group. J. Algebra 9, 220-239 (1968) MR 38:1991

[Sp] Spanier, E.: Algebraic Topology. New York: MacGraw-Hill 1966 MR 35:1007

[St1] Stanley, R. P.: Exponential structures. Stud. Appl. Math. 59, 73-82 (1978) MR $\mathbf{5 8 : 2 6 2}$

[St2] Stanley, R. P.: Some aspects of groups acting on finite posets. J. Combin. Theory Ser. A 32, 132-161 (1982) MR 83d:06002

[St3] Stanley, R. P.: Enumerative combinatorics I. Monterey, CA: Wadsworth \& Brooks / Cole, 1986 MR 87j:05003

[Su] Sundaram, S.: The homology representations of the symmetric group on CohenMacaulay subposets of the partition lattice. Adv. in Math. 104(2), 225-296 (1994) MR 94:11

[Su-Wa] Sundaram, S., Wachs, M.: The homology representations of the k-equal partition lattice. Trans. Amer. Math. Soc., to appear.

[tDi] tom Dieck, T.: Transformation Groups, de Gruyter, 1987. MR 89c:57048

[Va1] Vassiliev, V.A.: Cohomology of knot spaces. Advances in Soviet Math. 1, 23-69 (1990) MR 92a:57016

[Va2] Vassiliev, V.A.: Complements of discriminants of smooth maps : Topology and applications. (Transl. of Math. Monographs, Vol. 98). Providence, RI: Amer. Math. Soc. 1992 MR 94i:57020

[Va3] Vassiliev, V.A.: Complexes of connected graphs In: The Gelfand Seminar, 1990-1992, ed. Corwin, L., pp. 223-235, Basel, Boston: Birkhäuser 1993 MR 94h:55032

[Vo] Vogt, R.M.: Homotopy limits and colimits. Math. Zeitschrift. 134, 11-52 (1973) MR 48:12516 
[Wa] Wachs, M.: A basis for the homology of the d-divisible partition lattice. Adv. in Math. 117 (2), 294-318 (1996). CMP 96:07

[Zie-Ž] Ziegler, G. M., Živaljević, R.: Homotopy types of subspace arrangements via diagrams of spaces. Math. Annalen 295, 527-548 (1993) MR 94c:55018

Department of Mathematics and Computer Science, University of Miami, Coral Gables, Florida 33124

E-mail address: sheila@claude.math.wesleyan.edu

Current address: Department of Mathematics, Wesleyan University, Middletown, Connecticut 06459

Institute for Experimental Mathematics, Ellernstr. 29, 45326 Essen, Germany

E-mail address: welker@exp-math.uni-essen.de 